\title{
Long noncoding RNA MIR4435-2HG enhances metabolic function of myeloid dendritic cells from HIV-1 elite controllers
}

\author{
Ciputra Adijaya Hartana, ${ }^{1}$ Yelizaveta Rassadkina, ${ }^{1}$ Ce Gao, ${ }^{1}$ Enrique Martin-Gayo, ${ }^{2}$ Bruce D. Walker, ${ }^{1,3,4}$ Mathias Lichterfeld, ${ }^{1,5,6}$ \\ and Xu G. Yu ${ }^{1,5}$ \\ ${ }^{1}$ Ragon Institute of MGH, MIT and Harvard, Cambridge, Massachusetts, USA. ${ }^{2}$ Immunology Unit, Universidad Autónoma de Madrid, Hospital Universitario la Princesa, Madrid, Spain. ${ }^{3}$ Institute for Medical \\ Engineering and Sciences, and ${ }^{4}$ Department of Biology, Massachusetts Institute of Technology, Cambridge, Massachusetts, USA. ${ }^{5}$ Infectious Disease Division, Brigham and Women's Hospital, Boston, \\ Massachusetts, USA. ${ }^{6}$ Broad Institute of MIT and Harvard, Cambridge, Massachusetts, USA.
}

\begin{abstract}
Restriction of HIV-1 replication in elite controllers (ECs) is frequently attributed to T cell-mediated immune responses, while the specific contribution of innate immune cells is less clear. Here, we demonstrate an upregulation of the host long noncoding RNA (IncRNA) MIR4435-2HG in primary myeloid dendritic cells (mDCs) from ECs. Elevated expression of this IncRNA in mDCs was associated with a distinct immunometabolic profile, characterized by increased oxidative phosphorylation and glycolysis activities in response to TLR3 stimulation. Using functional assays, we show that MIR4435-2HC directly influenced the metabolic state of mDCs, likely through epigenetic mechanisms involving H3K27ac enrichment at an intronic enhancer in the RPTOR gene locus, the main component of the mammalian target of rapamycin complex 1 (mTORC1). Together, these results suggest a role of MIR4435-2HC for enhancing immunometabolic activities of mDCs in ECs through targeted epigenetic modifications of a member of the mTOR signaling pathway.
\end{abstract}

\section{Introduction}

Effective control of HIV-1 replication can be readily induced by highly active antiretroviral therapy (HAART) (1), but also occurs spontaneously in the absence of antiretroviral therapy in a small group of persons termed elite controllers (ECs) $(2,3)$. This extraordinary ability to control HIV-1 replication in the absence of pharmacological antiretroviral treatment provides a unique opportunity to study the dynamics of immune responses involved in restricting viral turnover. Indeed, recent studies suggest that immune responses in ECs are able to exert substantial selection pressure on virally infected cells, resulting in selective persistence of cells in which HIV-1 is integrated in repressive chromosomal regions, presumably conferring deep latency (4). Therefore, investigations of immune responses from ECs may provide clues for finding novel immunological targets for spontaneous control of HIV-1 infection (5).

Immune protection against HIV-1 disease progression has mostly been attributed to $\mathrm{T}$ cell-mediated immune responses, which can eliminate virally infected cells by HLA class I-restricted cytolysis and are frequently regarded as the cornerstone of spontaneous viral control in ECs (2). Dendritic cells (DCs) are innate professional antigen presenting cells and play critical roles in the induction of HIV-1specific T cell responses $(6,7)$. Upon HIV-1 sensing by endosomal and cytoplasmic microbial pattern recognition receptors, myeloid

Conflict of interest: The authors have declared that no conflict of interest exists. Copyright: (5) 2021, American Society for Clinical Investigation.

Submitted: November 16, 2020; Accepted: March 11, 2021; Published: May 3, 2021.

Reference information: J Clin Invest. 2021;131(9):e146136.

https://doi.org/10.1172/JCl146136.
DCs (mDCs) can prime, expand, and activate T cells. DCs are also reported to be permissive to productive HIV-1 infection (8), although their susceptibility to viral infection varies among DC subsets (9). Interestingly, mDCs from ECs frequently show elevated expression levels of immunomodulatory molecules (10), and seem to be endowed with improved abilities to mount cell-intrinsic type I interferon responses after recognition of HIV-1 (11). Such improved innate immune responses in $\mathrm{mDCs}$ are likely to critically contribute to the evolution of potent and effective cellular immune responses in ECs (12). The ability of mDCs from ECs to seemingly persist in an elevated functional modification state is etiologically unclear at present, but appears reminiscent of trained immunity, previously described in innate immune cells (13). The concept of trained immunity relates to improved functional responsiveness of innate immune cells due to epigenetic changes in immune profiles, frequently involving an altered metabolic activity $(14,15)$.

LncRNAs are defined as small, noncoding RNA species with nucleotides longer than 200 base pairs and have important regulatory roles for modulating functional states of cells (16). These effects are mediated through sequence-specific interactions of lncRNAs with other RNAs or with chromosomal DNA, and/or through molecular binding to paraspeckles and proteins $(17,18)$. Such interactions between lncRNAs and nucleic acids or proteins frequently modulate the position and function of chromatin-modifying enzymes, resulting in enhanced methylation or acetylation of the lysine constituents of histones or altered recruitment of transcription factors to specific chromosomal locations $(19,20)$. A role of lncRNAs in regulating the human immune response to a diverse range of pathogens is increasingly appreciated (21-23), and a possible function of 

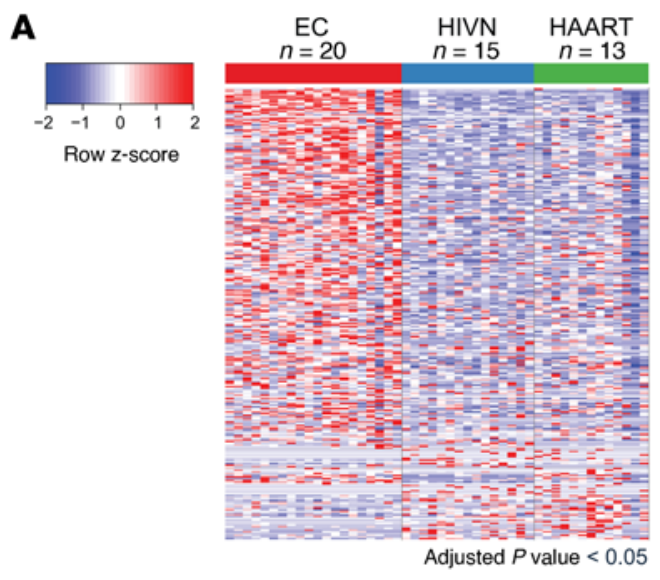

B

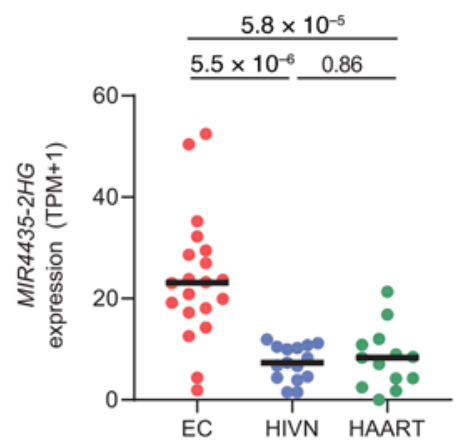

C

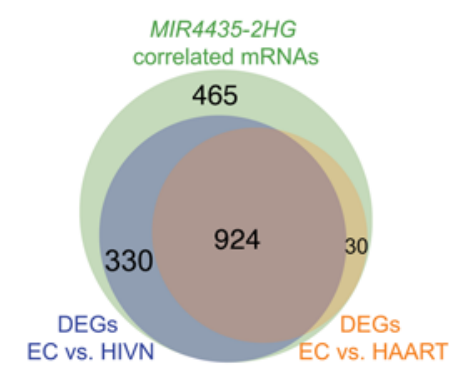

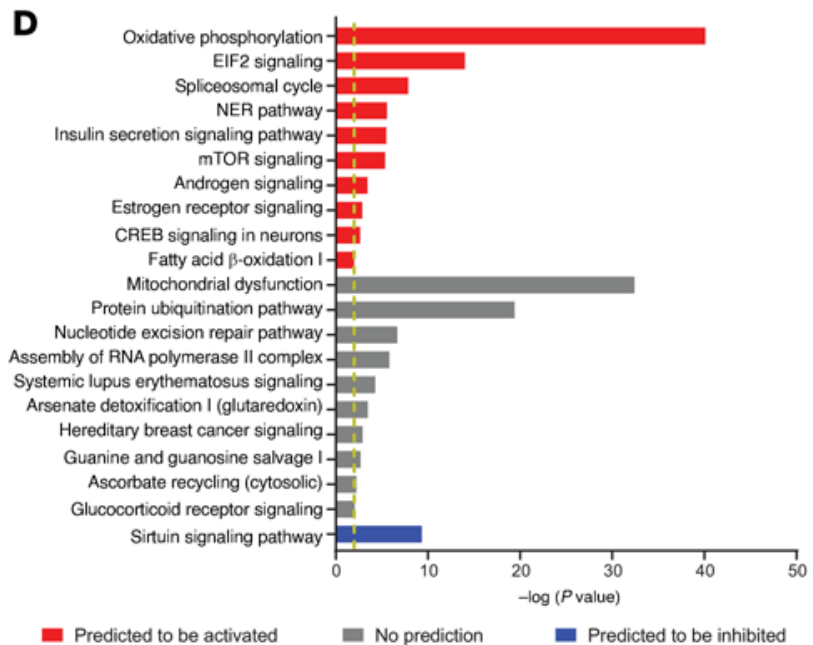

$\mathbf{E}$

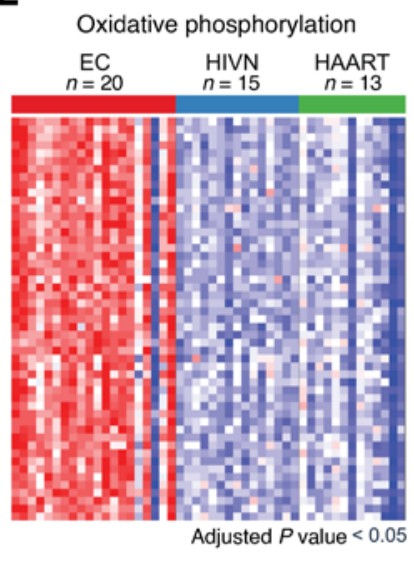

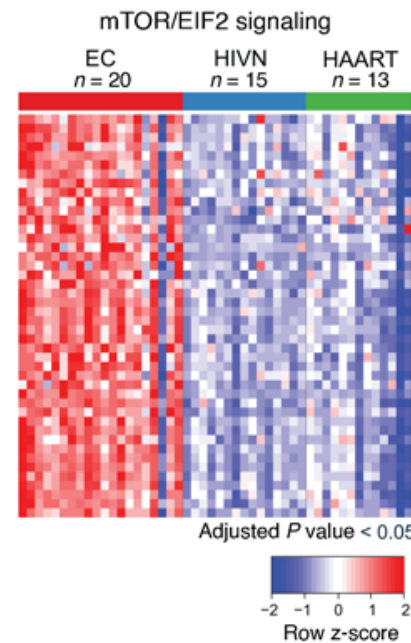

Figure 1. Increased expression of IncRNA MIR4435-2HC in primary mDCs from ECs. (A) Heatmap displaying differentially expressed IncRNAs (FDR-adjusted $P$ value $<0.05)$ in mDCs from ECs $(n=20)$ vs. HIVNs $(n=15)$ and HAARTs $(n=13)$ measured by RNAseq. (B) The expression $($ TPM+1) of MIR4435-2HG in mDCs was compared among ECs $(n=20)$, HIVNs $(n=15)$, and HAARTs $(n=13)$. Kruskal-Wallis test was used as the statistical test. (C) Venn diagram showing overlap between genes differentially expressed (FDR-adjusted $P$ value $<0.0001$ ) between EC vs. HIVN and EC vs. HAART and correlated with MIR4435-2HC expression (FDR-adjusted correlation $P$ value $<0.0001$ ). (D) Canonical pathways predicted to be significantly enriched for 924 DEGs from C, using IPA. Pathways predicated to be activated or inhibited are highlighted in red or blue, respectively. Pathways with no predicted change are marked in gray. Cutoff was established at $-\log (P$ value $) \geq 2$ (yellow dashed line) and $z$ score $>1.5$ or $<-1.5$. (E) Heatmaps displaying DEGs involved in oxidative phosphorylation or mTOR signaling predicted by IPA in $\mathbf{D}$ (FDR-adjusted $P$ value $<0.05$ ).

lncRNAs in modulating trained immunity of innate immune cells has been proposed by prior studies $(15,23,24)$. However, mechanistic insight into the activity of individual lncRNA species in the context of DCs and HIV-1 infection is currently scarce.

In this study, we observed a selective upregulation of lncRNA MIR4435-2HG in primary mDCs from ECs. Using complex immunometabolic profiling assays, we noted that MIR4435-2HG can activate oxidative phosphorylation in mDCs, most likely through epigenetic mechanisms involving H3K27ac enrichment at a recognized enhancer in the RPTOR gene locus, resulting in a higher transcription of this gene. Correspondingly, we found that RPTOR, the main component of the mammalian target of rapamycin complex 1 (mTORC1), was functionally controlling OXPHOS and glycolysis metabolisms in mDCs. Together, these results suggest a role for MIR4435-2HG in inducing an increased functional metabolic state in primary $\mathrm{mDCs}$ from ECs through targeted epigenetic modifications.

\section{Results}

Increased expression of IncRNA MIR4435-2HG in primary $m D C$ from ECs. LncRNAs may have important roles for regulating the transcriptional and functional behavior of mDCs during HIV-1 infection, but have never been systematically assessed in the rare subset of persons who naturally control HIV-1 replication in the absence of antiretroviral therapy (i.e., ECs). Here, we mined our previously generated RNAseq data (25) to evaluate lncRNA expressions in sorted mDCs (Supplemental Figure 1A; supplemental material available online with this article; https://doi.org/10.1172/JCI146136DS1) from a cohort of ECs $(n=20)$. HIV-1-negative healthy individuals (HIVNs; $n=15)$ and individuals treated with HAART (HAARTs; $n=13$ ) were analyzed as reference cohorts. The clinical and demographical characteristics of these study participants are summarized in Supplemental Table 1.

In total, we identified 55 and 8 lncRNAs that were significantly (FDR-adjusted $P$ value $<0.05$ ) up- or downregulated in ECs 

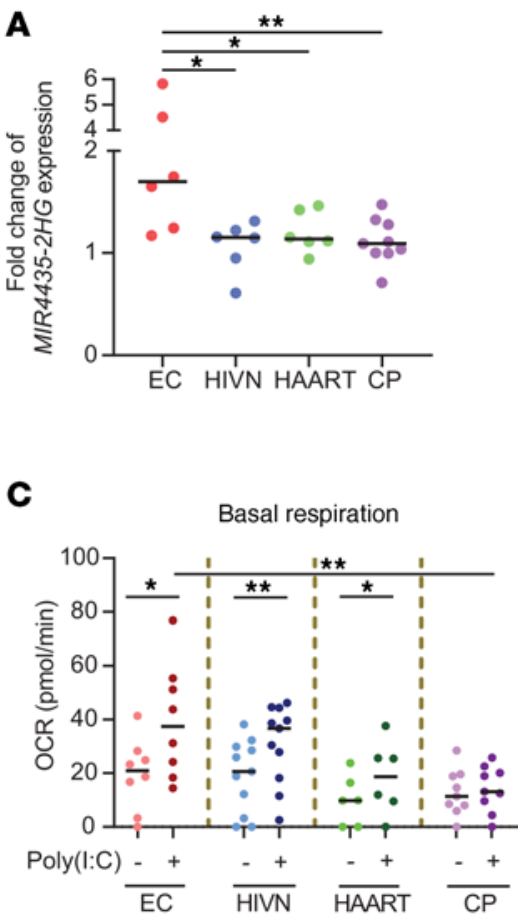
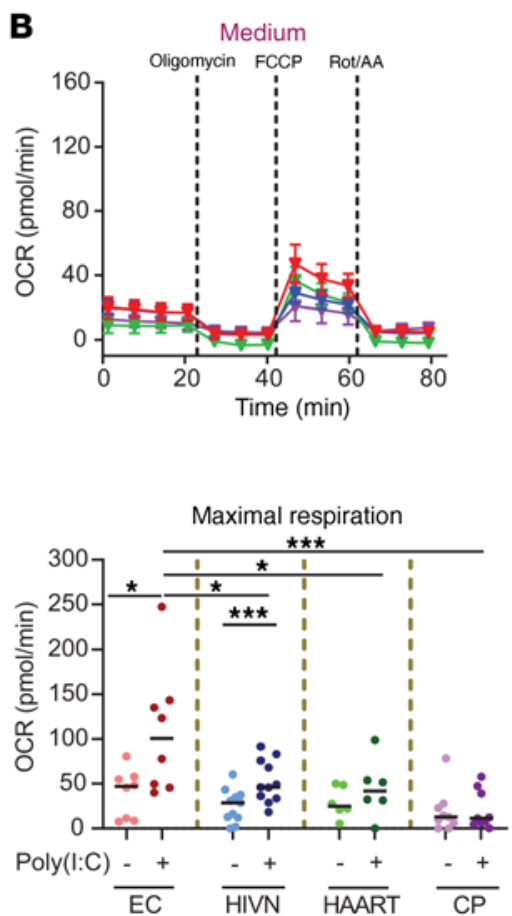
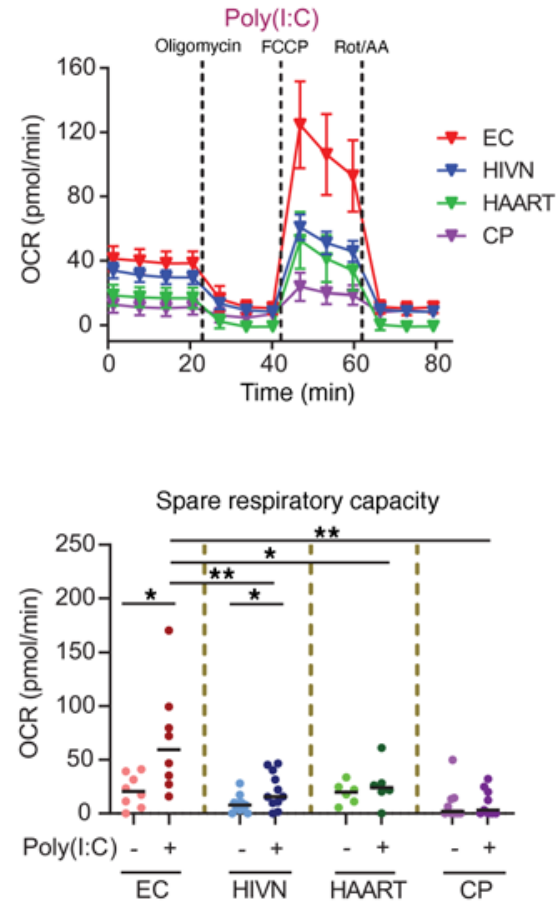

Figure 2. Upregulation of MIR4435-2HG in mDCs is associated with an increased oxidative phosphorylation. (A) The fold changes of MIR4435-2HG expression normalized to ACTB in mDCs from ECs $(n=6)$, HIVNs $(n=6)$, HAARTs $(n=6)$, and CPs $(n=9)$ after $2 \mu \mathrm{g} / \mathrm{mL}$ Poly(I:C) stimulation for 24 hours, relative to unstimulated control, was measured by RT-PCR. Kruskal-Wallis test was used for statistical analysis. (B) OCR of mDCs from ECs $(n=8)$, HIVNs $(n=11)$, HAARTs $(n=6)$, and CPs $(n=9)$ with or without $2 \mu \mathrm{g} / \mathrm{mL}$ Poly $(\mathrm{I}: \mathrm{C})$ stimulation for 24 hours was measured at indicated time points in responses to oligomycin, FCCP, and rotenone/antimycin A using a Seahorse XFe96 Analyzer. (C) Basal respiration, maximal respiration, and spare respiratory capacity in mDCs were compared in ECs, HIVNs, HAARTs, and CPs with or without $2 \mu \mathrm{g} / \mathrm{mL}$ Poly(I:C) stimulation for 24 hours. Paired, 2-tailed $t$ test was used to compare OCR between stimulated and unstimulated cells, whereas 1-way ANOVA test was used to compare OCR among cohorts. ${ }^{*} P<0.05,{ }^{* *} P<0.01,{ }^{* * *} P<0.001,{ }^{* * * *} P<0.0001$.

compared with both reference cohorts (Figure 1A and Supplemental Figure 1B). Subsequent filtering according to more robust statistical criteria (Supplemental Figure 1B) allowed us to detect 4 upregulated lncRNAs for which differences between ECs and reference cohorts were most evident. All of these 4 lncRNAs were more strongly expressed in mDCs, but not in monocytes, from ECs compared with HIVNs and HAARTs (Figure 1B and Supplemental Figure 1, C and D). Out of these 4 lncRNA transcripts, lncRNA MIR4435-2HG was selected for further study due to its possible functional significance as a regulator of immune cell survival and activation in alternative infectious disease contexts $(26,27)$.

To predict the biological regulatory functions of MIR4435$2 \mathrm{HG}$ on gene expression signatures from $\mathrm{mDCs}$, we performed a correlation analysis between expression intensities of the lncRNA and mRNAs in primary mDCs from the 3 different study cohorts. We observed that out of a total of 1749 transcripts that were strongly associated with MIR4435-2HG (FDR-adjusted $P$ value < 0.0001), 924 genes were differentially expressed between ECs and either one of the 2 control cohorts (FDR-adjusted $P$ value $<$ 0.0001) (Figure 1C). Ingenuity pathway analysis (IPA) (28) suggested activation of oxidative phosphorylation (OXPHOS) as the predominant canonical pathway that these 924 transcripts were involved in (Figure 1D). OXPHOS was also identified as the major underlying functional activity of these genes using the DAVID gene ontology prediction tool (Supplemental Figure 2A) $(29,30)$. Notably, other metabolic pathways known to support OXPHOS, such as the mTOR signaling pathway and the $\beta$-oxidation of fatty acids, were also predicted to be activated by these transcripts, as well as the mTOR-associated EIF2 signaling pathway (Figure 1D) $(31,32)$. Correspondingly, a list of transcripts known to be involved in OXPHOS and mTOR/EIF2 pathways met criteria for differential expression between ECs and other study cohorts (Figure 1E). Therefore, we hypothesized that upregulation of MIR4435-2HG may alter the oxidative metabolism and affect ensuing immunometabolic functions of mDCs from ECs.

Upregulation of MIR4435-2HG in $\mathrm{MDCs}$ is associated with a distinct metabolic profile. To evaluate possible functional consequences of MIR4435-2HG upregulation in mDCs from ECs, we performed an evaluation of the metabolic profile of these cells, relative to control cells from HIVNs, HAARTs, and viremic HIV-1infected individuals not treated with HAART (chronic progressors, CPs). For this purpose, we stimulated mDCs from these cohorts using Poly(I:C). Higher MIR4435-2HG expression remained clearly visible in ECs after stimulation with Poly(I:C) (Figure 2A). Subsequently, we measured OXPHOS activities by means of oxygen consumption rates (OCRs), using the Seahorse technology (33). Following activation, we observed significant increases of basal respiration, maximal respiration, and spare respiratory capacity in primary mDCs from both ECs $(n=8)$ and HIVNs $(n=11)$, while such changes were less pronounced in HAARTs $(n=6)$ and CPs $(n=9)$ (Figure 2, B and C). Interestingly, maximal respiration and spare respiratory capacity were significantly higher in $\mathrm{mDCs}$ from 

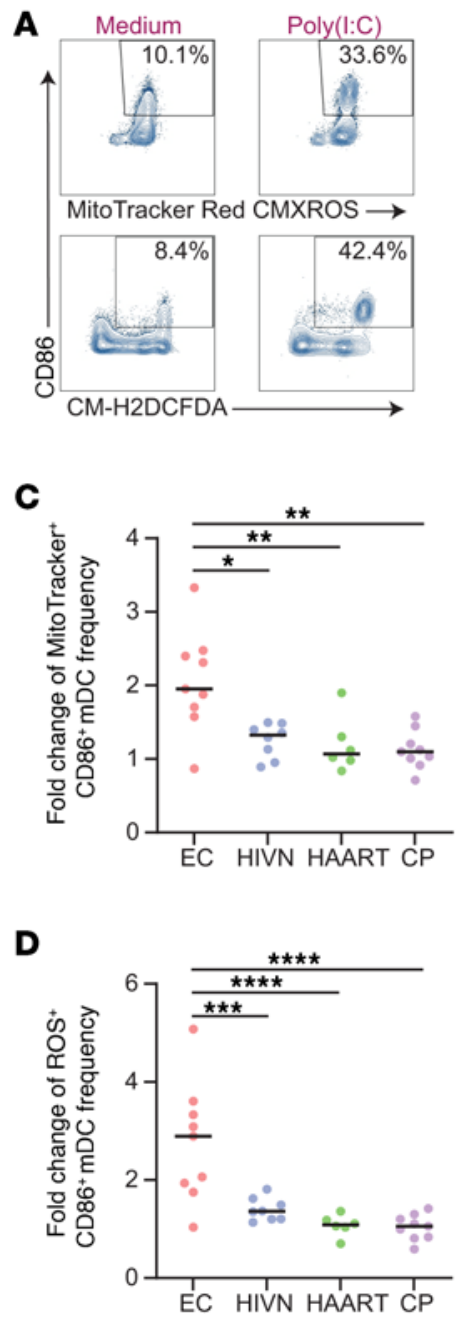
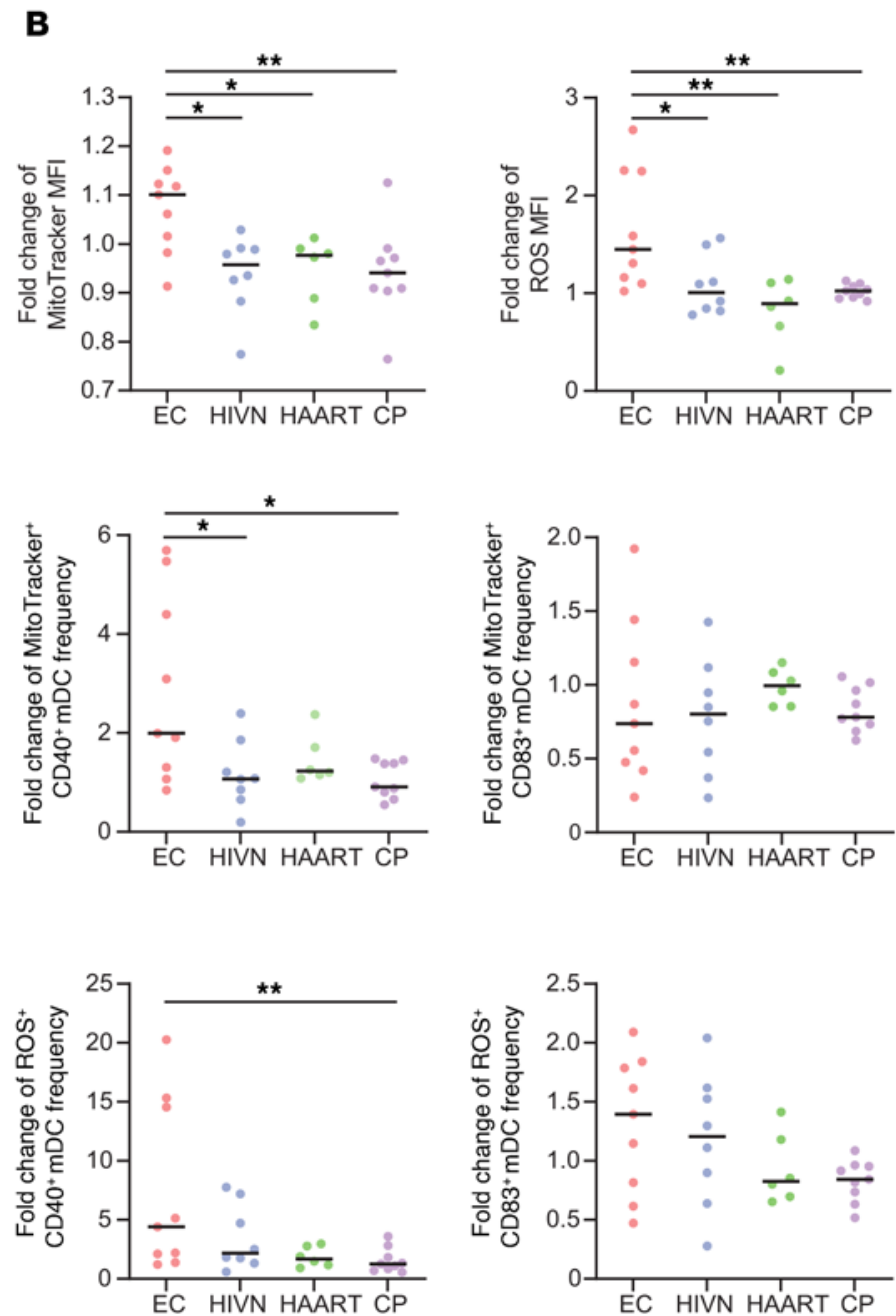

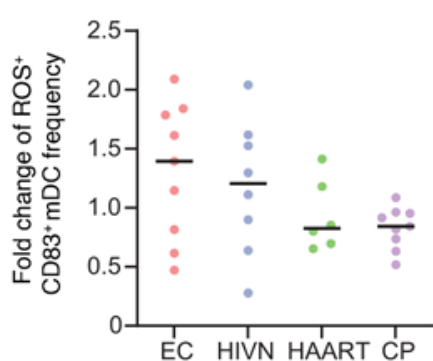

Figure 3. Upregulation of MIR4435-2HG in mDCs is associated with an enhanced metabolic profile. (A) Flow cytometry contour plots displaying the coexpression of MitoTracker or ROS and activation marker CD86 in mDCs from a representative EC with or without $2 \mu \mathrm{g} / \mathrm{mL}$ Poly(I:C) stimulation for 24 hours. (B) The MFIs of MitoTracker and ROS in total mDCs were compared among ECs $(n=9)$, HIVNs $(n=8)$, HAARTs $(n=6)$, and CPs $(n=9)$. The MFIs were shown as fold changes after Poly(l:C) stimulation, relative to unstimulated controls. Kruskal-Wallis test was used for statistical analysis. (C, D) The frequencies of mDCs coexpressing MitoTracker or ROS and activation markers (CD86, CD40, and CD83) in ECs $(n=9)$, HIVNs $(n=8)$, HAARTs $(n=6)$, and CPs $(n=9)$ were compared. The frequencies were expressed as fold changes of respective cell populations after Poly(l:C) stimulation, relative to unstimulated conditions. Kruskal-Wallis test was used to compare the fold changes of cell frequencies among cohorts. ${ }^{*} P<0.05,{ }^{* *} P<0.01,{ }^{* * *} P<0.001,{ }^{* * * *} P<0.0001$.

ECs compared with all 3 reference cohorts after Poly(I:C) stimulation (Figure 2C), highlighting remarkably elevated OXPHOS activities in $\mathrm{mDCs}$ from ECs.

Mitochondrial OXPHOS is known to be associated with an increased membrane polarization in mitochondria and production of reactive oxygen species (ROS), which can be flow-cytometrically assessed using MitoTracker Red CMXROS and CM-H2DCFDA dyes, respectively (Figure 3A). We observed that after Poly(I:C) stimulation, mDCs from ECs displayed increased mitochondrial polarization and ROS production (Figure $3 \mathrm{~B}$ ), specifically in the subset of activated mDCs expressing the costimulatory molecule CD86 (Figure 3, A, C, and D). The increase in the frequencies of MitoTracker ${ }^{+} \mathrm{CD} 86^{+} \mathrm{mDC}$ following Poly(I:C) stimulation was significantly higher in ECs relative to control cells from HIVNs, HAARTs, and CPs $(P=0.0249, P=0.0091$, and $P=0.0026$, respectively) (Figure $3 C$ ). In addition, the proportion of MitoTracker ${ }^{+}$ $\mathrm{CD}_{40} \mathrm{O}^{+} \mathrm{mDC}$ was significantly higher in ECs relative to HIVNs and
CPs $(P=0.0214$ and $P=0.0138$, respectively $)$, while no differences were noted between MitoTracker ${ }^{+} \mathrm{CD} 83^{+} \mathrm{mDCs}$ among all cohorts (Figure 3C). Notably, we also observed a significant increase of $\mathrm{ROS}^{+} \mathrm{CD} 86^{+} \mathrm{mDC}$ frequencies after Poly(I:C) stimulation from ECs compared with all the other cohorts (all $P<0.001$ ) (Figure 3D). ROS expression in $\mathrm{CD} 4 \mathrm{O}^{+} \mathrm{mDCs}$ was higher in ECs compared with CPs $(P=0.0072)$, but was indistinguishable in $\mathrm{CD}^{2} 3^{+} \mathrm{mDCs}$ between poly(I:C)-stimulated mDCs from all cohorts (Figure 3D). Moreover, we noted positive correlations between MIR443-2HG expression and the frequencies of MitoTracker ${ }^{+} \mathrm{CD}^{+} 6^{+}$and $\mathrm{ROS}^{+}$ $\mathrm{CD}^{+} 6^{+} \mathrm{mDCs}$ in ECs (Supplemental Figure 3, A and B).

To extend these observations, we measured the extracellular acidification rate (ECAR), an established correlate of cellular glycolytic activity, in Poly(I:C)-stimulated mDCs. Poly(I:C) stimulation increased the ECAR in mDCs from ECs and HIVNs, and this effect was significantly more pronounced in the ECs compared with HIVNs, HAARTs, and CPs $(P=0.0041, P<0.0001$, and $P<0.0001$, 

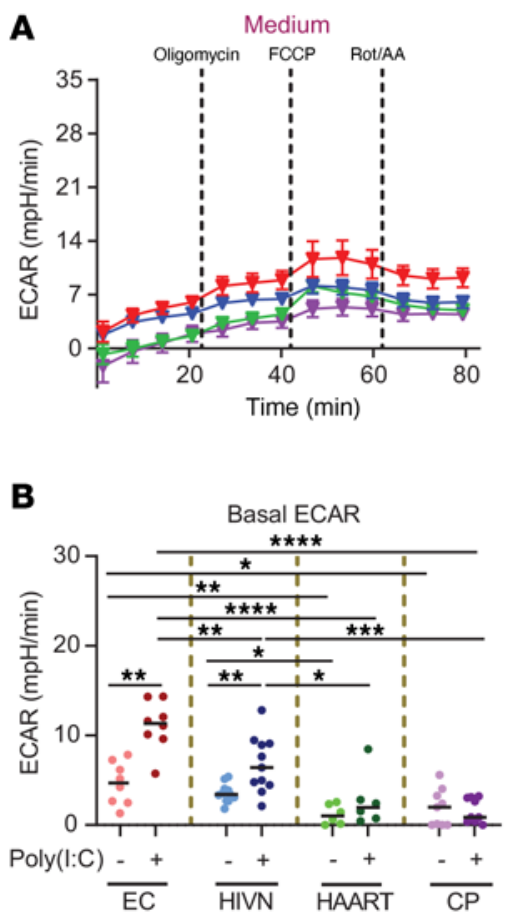
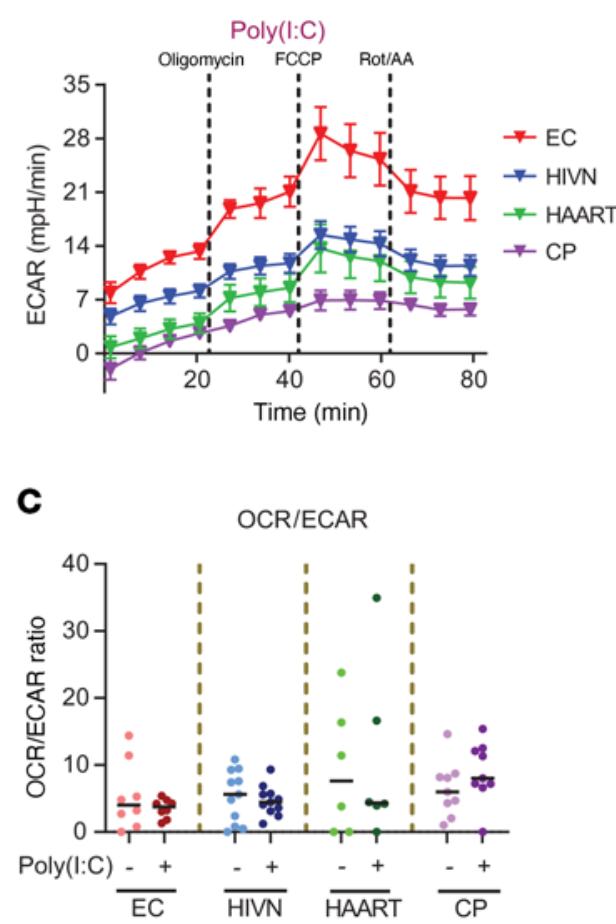

Figure 4. Upregulation of MIR4435-2HC in $\mathrm{mDCs}$ is associated with increased glycolysis. (A) Extracellular acidification rate (ECAR) of $\mathrm{mDCs}$ from Figure 2B was measured using a Seahorse XFe96 Analyzer. (B) Basal ECAR in $\mathrm{mDC}$ from study population in $\mathbf{A}$ was compared. Paired, 2-tailed $t$ test was used to compare ECARs between stimulated and unstimulated cells, whereas 1-way ANOVA test was used to compare ECARs among cohorts. (C) Intraindividual comparisons between basal OCR normalized to basal ECAR. Paired, 2-tailed $t$ test was used to compare OCR/ECAR ratio between stimulations, whereas 1-way ANOVA test was used to compare OCR/ECAR among cohorts. ${ }^{*} P<0.05,{ }^{*} P<0.01$, ${ }^{* * *} P<0.001,{ }^{* * *} P<0.0001$ respectively) (Figure 4, A and B). Notably, no changes in OCR/ECAR ratios were seen following Poly(I:C) stimulation in all study cohorts, suggesting that both OXPHOS and glycolysis are induced in parallel (Figure 4C). Taken together, these results suggest that upregulation of MIR4435-2HG in mDCs from ECs is associated with a distinct functional metabolic profile, characterized by increases of both OXPHOS and glycolytic activities following Poly(I:C) stimulation.

MIR4435-2HG is expressed in subsets of mDCs with phenotypic features of increased activation and function. To better characterize the expression of MIR4435-2HG in defined mDC subpopulations, we optimized a PrimeFlow RNA detection assay for evaluating lncRNA MIR 4435-2HG expression directly ex vivo in primary $\mathrm{mDCs}$ by flow cytometry. First, using this flow cytometry-based detection technique, we confirmed that the expression of MIR4435-2HG is higher in mDCs from ECs $(n=8)$ than in those from HIVNs $(n=$ 13) $(P=0.0103)$ (Figure $5 \mathrm{~A})$, while no difference in $\mathrm{mDC}$ frequencies between ECs and HIVNs was detected (Supplemental Figure 4A). Moreover, MIR4435-2HG was not differentially expressed between plasmacytoid dendritic cells (pDCs) or natural killer (NK) cells from the 2 study cohorts (Supplemental Figure 4B). Expression of MIR4435-2HG in total mDCs was positively associated with the expression of the costimulatory molecule CD86 (Figure 5B), and the mean fluorescence intensity (MFI) of $M I R 4435-2 H G$ in $\mathrm{CD}^{2} 6^{+}$ mDCs was significantly higher compared with CD86 ${ }^{-}$mDCs, both in ECs and in HIVNs (Figure 5C). In addition, a positive association was seen between MIR4435-2HG and the immunoregulatory receptor LILRB2 (Figure 5B and Supplemental Figure 4C). However, MIR4435-2HG expression was not significantly different between LILRB2 $^{+}$mDCs from ECs and HIVNs (Supplemental Figure 4D). We subsequently used t-distributed stochastic neighbor embedding (tSNE) and flow self-organizing maps (FlowSOM) (34) to identify phenotypically defined mDC subpopulations (Supplemental Figure 1A). FlowSOM identified 7 major, phenotypically distinct mDC for these subsets did not differ among the 2 study cohorts (Figure 6, E and F). Notably, we observed that $\mathrm{CD} 16^{+} \mathrm{mDCs}$, a cell subset endowed with increased abilities for antigen presentation, and previously classified as the DC4 subpopulation (35) or as CD16 ${ }^{+} \mathrm{CD} 14^{\mathrm{dim}}$ nonclassical monocytes (36-38), were subdivided by FlowSOM into 2 different clusters (clusters 2 and 7) (Figure 6A). Cluster 2 mDCs exhibited moderate expression intensity of MIR4435-2HG (Figure $6, \mathrm{~B}$ and $\mathrm{C}$ ) and were markedly increased in ECs, accounting for more than $60 \%$ of all mDCs in ECs and less than $25 \%$ of mDCs in HIVNs $(P=0.0006)$ (Figure 6D). In contrast, cluster $7 \mathrm{mDCs}$ exhibited low/absent expression of MIR4435-2HG (Figure 6, B and C) and was greatly expanded in HIVNs compared with ECs $(29.18 \%$ vs. $0.09 \%, P=0.0112$ ) (Figure 6D). Finally, cluster 3, a CD141 ${ }^{+} \mathrm{mDC}$ cluster, which is reminiscent of the cross-presenting DC1 cell subpopulation described previously (35), showed a significantly higher per-cell expression of MIR4435-2HG in ECs compared with HIVNs (Figure 6E). However, the frequency of this cluster was small ( 1\% in both cohorts) (Figure 6D). Together, these studies demonstrate that upregulation of MIR4435-2HG in mDCs from ECs is due to (a) expansion of subsets with moderate MIR4435-2HG expression (cluster 2), (b) reduction of subsets with low MIR4435-2HG expression (cluster 7), and (c) an increase in per-cell levels of MIR4435$2 \mathrm{HG}$ expression in cluster 3 (Figure 6, B-F). We also observed that in clusters 2, 3, 4, and 5, MIR4435-2HG correlated positively with various mDC activation markers, in contrast to the MIR4435-2HG-low/ absent cluster 7 (Supplemental Figure 5B). Therefore, the selective upregulation of MIR4425-2HG in mDCs with phenotypic features of more advanced maturation suggests an involvement of this lncRNA in regulating the functional profile of $\mathrm{mDCs}$.

MIR4435-2HG modulates functional metabolic activities in mDCs. Based on the close associations between MIR4435-2HG expression and the functional metabolic profiles of mDCs from ECs, in particular the OXPHOS and mTOR signaling pathways (Figure 

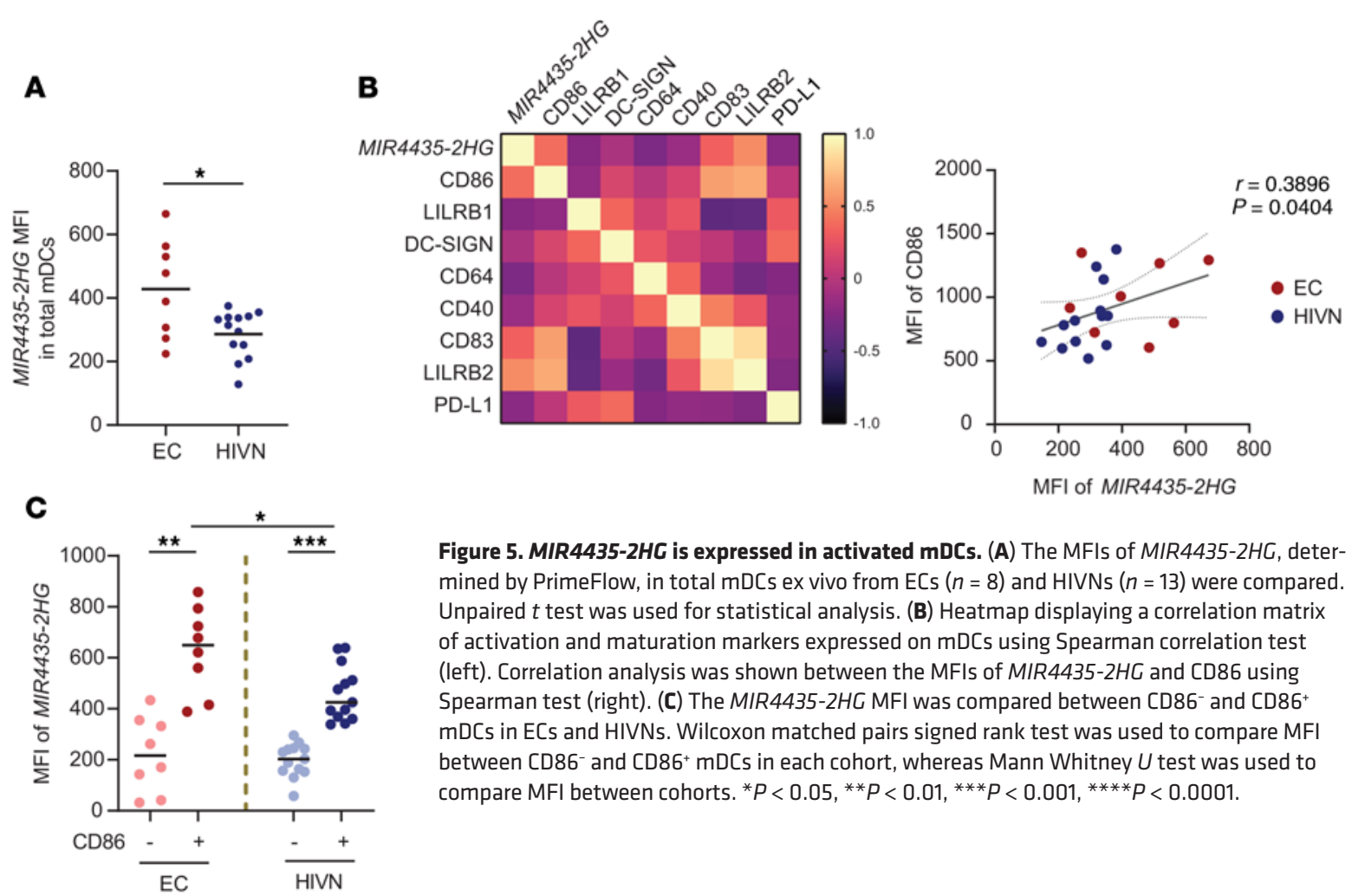

\begin{abstract}
Figure 5. MIR4435-2HC is expressed in activated mDCs. (A) The MFIs of MIR4435-2HG, determined by PrimeFlow, in total mDCs ex vivo from ECs $(n=8)$ and HIVNs $(n=13)$ were compared. Unpaired $t$ test was used for statistical analysis. (B) Heatmap displaying a correlation matrix of activation and maturation markers expressed on mDCs using Spearman correlation test (left). Correlation analysis was shown between the MFIs of MIR4435-2HG and CD86 using Spearman test (right). (C) The MIR4435-2HG MFI was compared between CD86- and CD86+ mDCs in ECs and HIVNs. Wilcoxon matched pairs signed rank test was used to compare MFI between $\mathrm{CD}^{-} 6^{-}$and $\mathrm{CD} 86^{+} \mathrm{mDCs}$ in each cohort, whereas Mann Whitney $U$ test was used to compare MFI between cohorts. ${ }^{*} P<0.05,{ }^{* *} P<0.01,{ }^{* *} P<0.001,{ }^{* * *} P<0.0001$.
\end{abstract}

$1, \mathrm{C}$ and $\mathrm{D})$, we subsequently conducted in vitro experiments to directly evaluate the possible regulatory roles of this lncRNA on host transcriptional and metabolic activity. To address this, mDCs from HIVNs $(n=5)$ were isolated and subjected to siRNA-mediated gene silencing of MIR4435-2HG, followed by Poly(I:C) stimulation (Figure 7A). SiRNA transfection allowed for an efficient downregulation of MIR4435-2HG, relative to a nonspecific scramble siRNA (Supplemental Figure 6A). Following MIR4435-2HG knockdown, we observed significant changes in gene expression in Poly(I:C)-activated mDCs, with 1035 genes meeting criteria for differential expression (FDR-adjusted $P$ value $<0.05$ ) (Figure 7B). IPA of these differentially expressed genes predicted a functional inhibition of canonical pathways related to OXPHOS, TNFR2, and TNFR1 signaling, mTOR/EIF2 signaling, and production of nitric oxide (NO) and ROS following downregulation of MIR4435-2HG (Figure 7C). Notably, the apparent repression of OXPHOS and the mTOR/ EIF2 pathways (Supplemental Figure 6B) following experimental MIR4435-2HG downregulation resonates with the activation of these pathways in mDCs from ECs (Figure 1D and Supplemental Figure 6C), in which we observed higher expression levels of MIR4435-2HG. Therefore, these results suggest that MIR4335-2HG upregulation in $\mathrm{mDCs}$ from ECs plays a critical role for regulating and inducing increased metabolic activities in these cells.

To further explore functional effects of MIR4435-2HG, we measured OXPHOS and glycolytic activity in mDCs after MIR4435-2HG gene silencing (Figure 7A). As described above, MIR4435-2HGspecific and scramble control siRNA were nucleofected into primary mDCs, with efficient lncRNA silencing and uncompromised viability of mDCs (Supplemental Figure 6, D and E), followed by stimulation with Poly(I:C). We noted that the basal respiration and maximal respiration were significantly lower when MIR4435-2HG was silenced ( $P=0.0078$ and $P=0.0078$, respectively), while the spare respiratory capacity seemed to be unaffected (Figure 8 , A and B). Notably, MIR4435-2HG gene silencing did not seem to influence the baseline ECAR or the OCR/ECAR ratio (Figure 8, A and C). However, increases in ECAR following experimental blockade of respiratory chain complexes by oligomycin and Rotenone/Antimycin A were less pronounced in mDCs treated with $M I R 4435-2 H^{-}$ specific siRNA (Figure 8A); this suggests that silencing of MIR4435$2 H G$ weakens the cellular ability to upregulate nonoxidative glycolytic activity when oxidative phosphorylation is inhibited. The frequency of CD40-, CD83-, and CD86-expressing mDCs coexpressing MitoTracker or ROS was significantly reduced exclusively following Poly(I:C) stimulation (Supplemental Figure 7, A and B) once MIR4435-2HG was silenced (Figure 8, D-F), further indicating lower OXPHOS and costimulation activities after inhibition of MIR4435-2HG. Consequently, when MIR4435-2HG was silenced, the ability of mDCs to induce $\mathrm{CD} 4^{+}$and $\mathrm{CD} 8^{+} \mathrm{T}$ cell proliferation was significantly reduced $(P=0.0313$ and $P=0.0469$, respectively) (Figure $8, G$ and $H$ ). In combination with the transcriptional changes reported above (Figure 7, B and C), these functional assays support the hypothesis that $M I R 4435-2 H G$ is critically involved in regulating the metabolic activities of $\mathrm{mDCs}$.

MIR4435-2HG influences RPTOR expression in mDCs through H3K27ac histone modification. LncRNAs can modify gene expression by epigenetic alterations through the recruitment of inhibitory or activating histone-modifying enzymes to defined chromosomal DNA locations $(19,20,39)$. One proposed mechanism responsible for such regulatory functions of lncRNAs is the formation of triple helices, characterized by insertion of the IncRNA into the major groove of the duplex DNA structure; such a triple-helix configuration is enabled by sequence-specific interactions between DNA-binding 
A

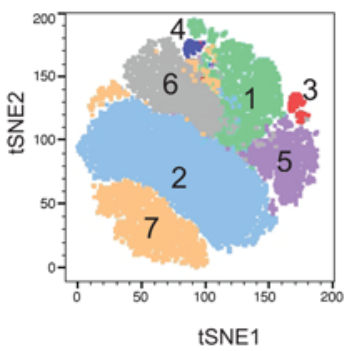

C
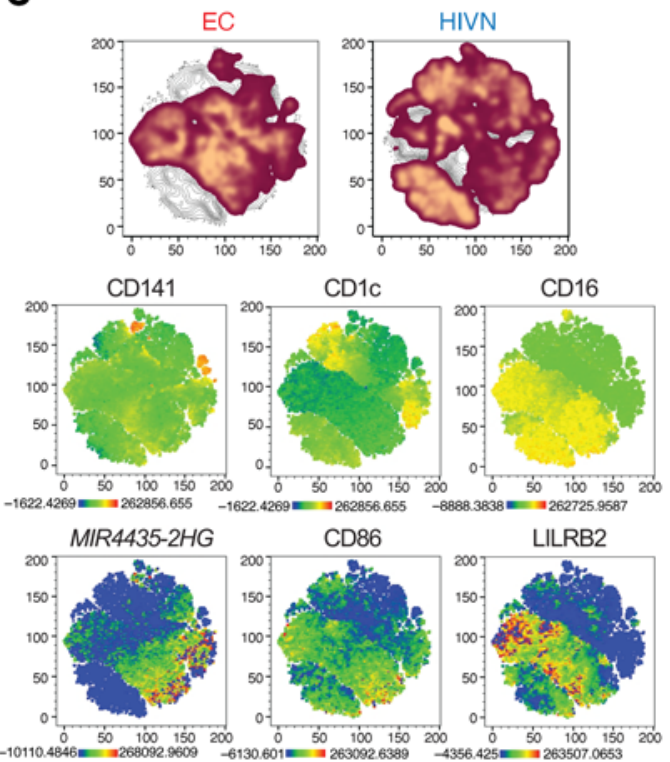

CD40
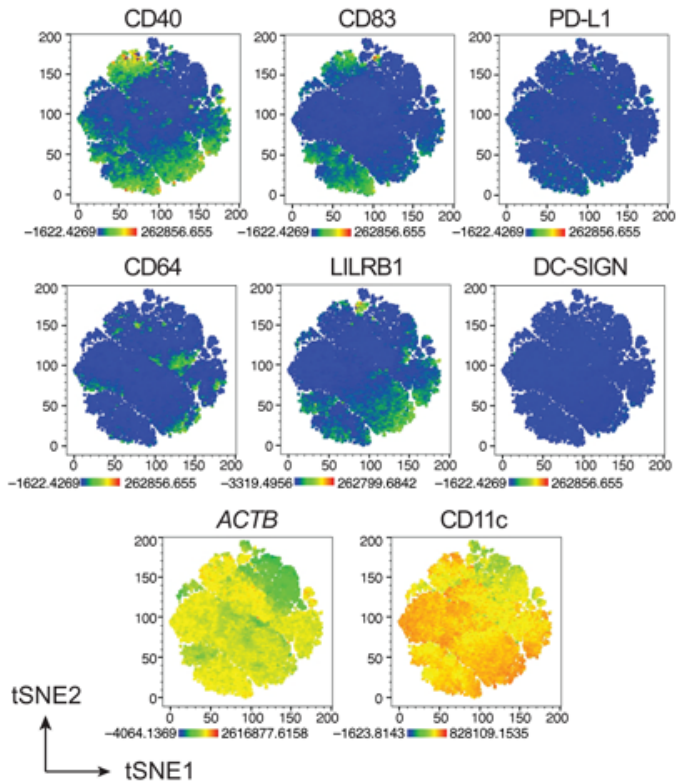

B

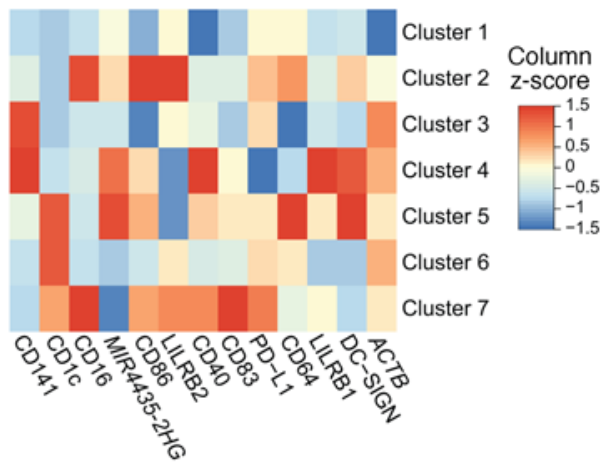

D

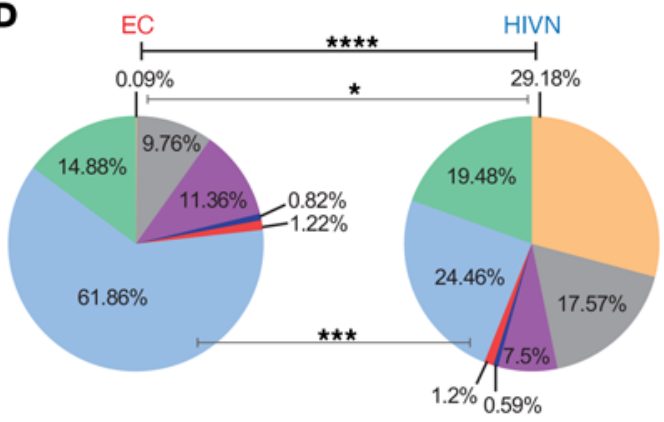

$$
\begin{aligned}
& \text { - Cluster } 1=\text { Cluster } 2=\text { Cluster } 3=\text { Cluster } 4 \\
& \text { - Cluster } 5=\text { Cluster } 6=\text { Cluster } 7
\end{aligned}
$$

$\mathbf{E}$
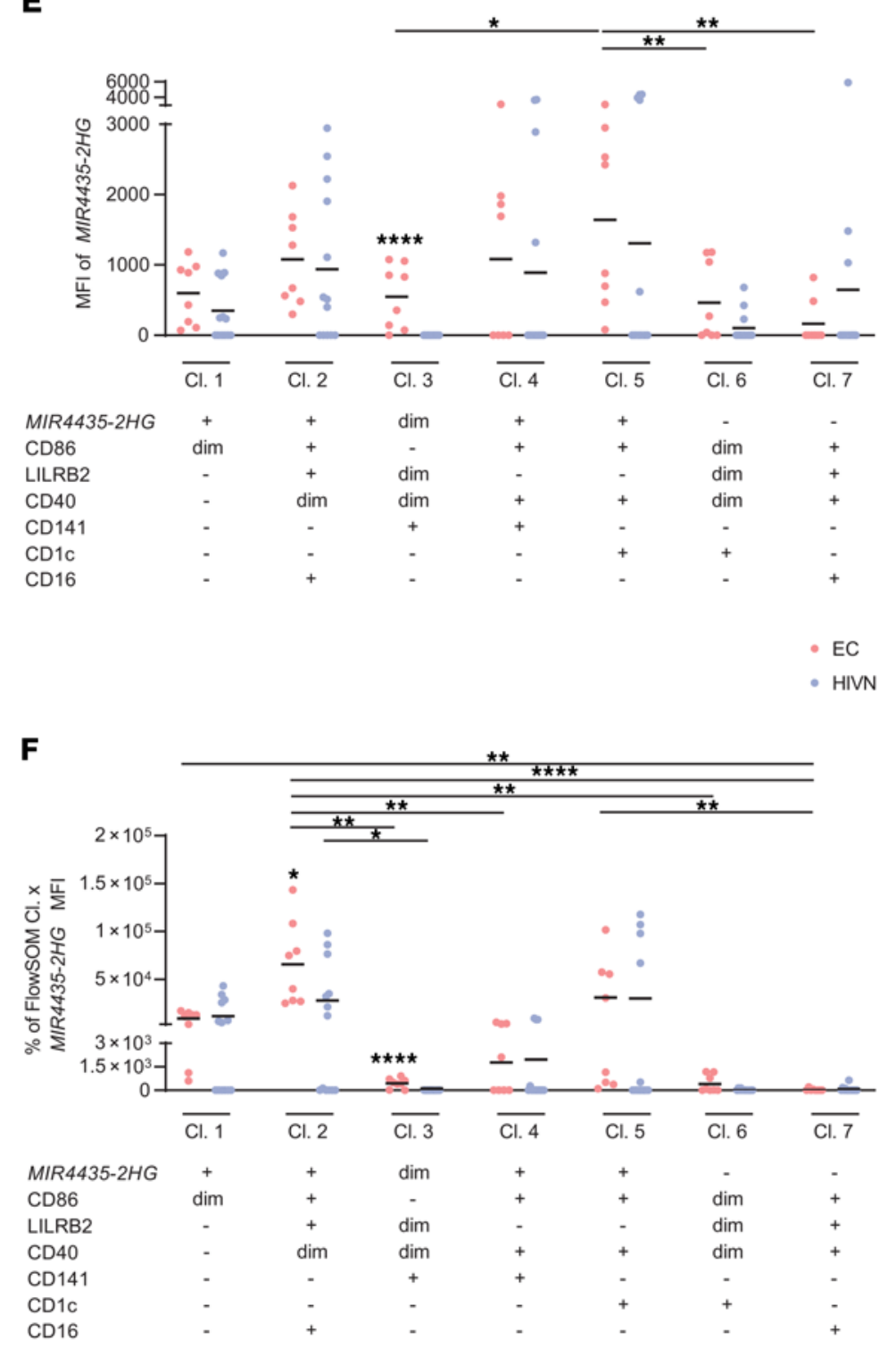

Figure 6. MIR4435-2HC is expressed in subsets of mDCs with phenotypic features of increased activation and function. (A) tSNE map displaying the concatenated mDCs from ECs $(n=8)$ and HIVNs $(n=13)$ in 7 clusters identified by FlowSOM. (B) Heatmap showing the MFI of the parameters measured in the 7 mDC clusters from A. (C) Global tSNE map of concatenated mDCs, with mDCs from ECs and HIVNs overlaid (top). tSNE map showing the expression of individual phenotypic markers measured by flow cytometry (bottom). (D) Pie charts displaying the average frequencies of the 7 clusters in each cohort. Mann Whitney $U$ test was used to compare the frequencies of each cluster between ECs and HIVNs, whereas $\chi^{2}$ test was used to compare the overall frequency distribution. (E) The MFIs of MIR4435-2HG were compared among 7 clusters in ECs and HIVNs. Mann Whitney $U$ test was used for comparison between ECs and HIVNs, whereas Friedman test was used to compare among clusters. (F) The multiplication product of cluster frequencies (D) and MIR4435-2HG MFI (E) was calculated as a composite read-out and compared as in $\mathbf{E} .{ }^{*} P<0.05,{ }^{* *} P<0.01,{ }^{* *} P<0.001,{ }^{* * *} P<0.0001$. 
A

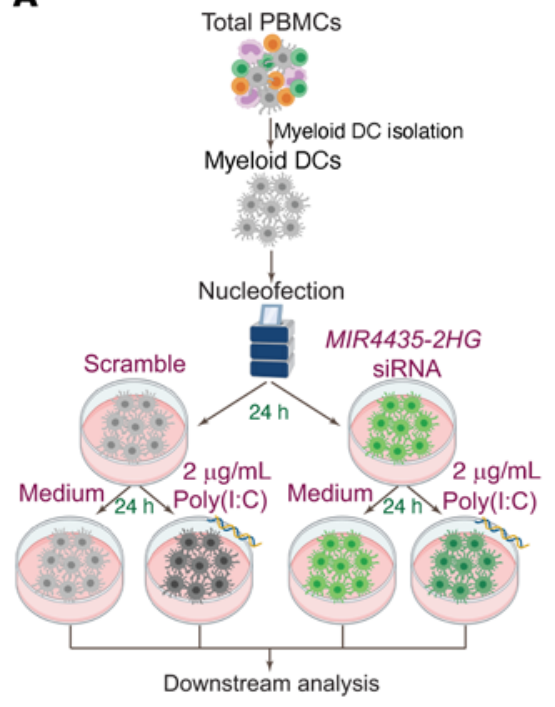

B

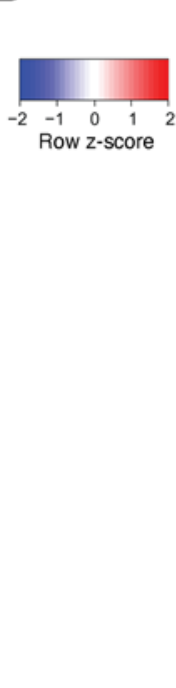

C

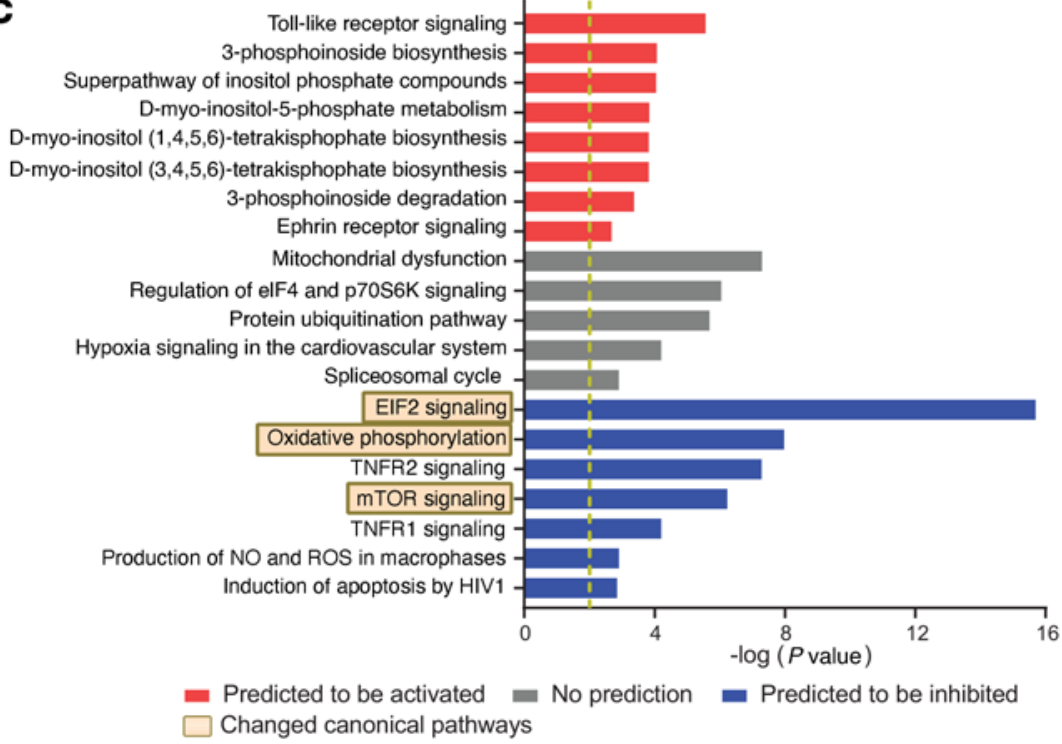

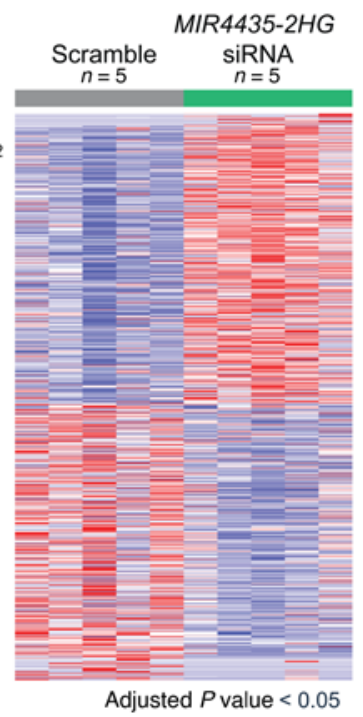

Adjusted $P$ value $<0.05$ BaseMean $>1$

Notably, the RPTOR gene encodes for a major component of the mTOR subcomplex mTORC1 and was downregulated following experimental silencing of MIR4435-2HG (Figure 7, B and C, and Figure 9C). Epigenetic changes at this particular genomic site were specific for H3K27ac, whereas H3K27me3 and H3K4me3 signal intensity at this gene locus was not different between the 2 study cohorts (Figure 9, D and E). Corresponding to these findings, we noted a significant positive correlation between IncRNA MIR4435-2HG expression and RPTOR mRNA expression in ECs (Figure 9F). Moreover, downregulation of RPTOR mRNA (Figure 9G) and protein (Figure $9 \mathrm{H}$ ) expression was noted after experimental MIR4435-2HG gene silencing by siRNA in ECs. Collectively, these results suggest that MIR4435-2HG can effectively increase RPTOR gene expression through triple helix formation at an intronic RPTOR gene enhancer, with resulting enrichment of the activating chromatin marker H3K27ac to this genomic location, likely through specific recruitment of histone acetyltransferases.

mTORC1 inhibition reduced OXPHOS,

domains of lncRNAs and specific chromosomal DNA regions (40). To evaluate epigenetic effects of MIR4435-2HG on chromatin structure and histone modifications, we performed cleavage under target $\&$ release using nuclease (CUT\&RUN) assay in mDCs from ECs ( $n$ $=4)$ and HIVNs $(n=4)$, allowing for identification of DNA segments binding to defined activating and inhibitory histone marks (41). These experiments demonstrated a significant enrichment or deenrichment of H3K27ac-, H3K27me3-, and H3K4me3-associated DNA segments at multiple genomic loci in ECs relative to HIVNs (Figure 9A, Supplemental Figure 8, A and B, and Supplemental Table 2), suggesting considerable differences in epigenetic regulation between mDCs from the 2 study cohorts. Notably, we observed significantly increased H3K27ac enrichment at an intronic enhancer region within the RPTOR gene in mDCs from ECs compared with HIVNs (Figure 9B), a genomic locus for which computational algorithms (40) specifically predicted susceptibility to triple-helix formation between chromosomal double-stranded DNA and MIR4435$2 H G$ using the triplex domain finder (TDF) algorithm (Figure 9C). glycolysis, and functional activities in mDCs. RPTOR is the main functional component of mTORC1, a major subcomponent of the mTOR signaling pathway which regulates cellular metabolism and activation in DCs and other cells (42). We hypothesized that MIR4435-2HG increases the immunometabolic activity of mDCs through enhanced epigenetic modification of H3K27ac in the RPTOR gene locus, resulting in increased RPTOR gene expression and activation of the mTORC1 complex. To test this, we analyzed the functional and metabolic profile of mDCs in the presence or absence of rapamycin treatment. We noted that the mTORC1 inhibitor rapamycin significantly reduced basal respiration, maximal respiration, and spare respiratory capacity in Poly(I:C)-stimulated mDCs (Figure 10, A and B). In addition, rapamycin also inhibited glycolysis, as lower extracellular acidification rate levels were observed following addition of rapamycin, without detectable differences in the OCR/ECAR ratio (Figure 10, A and C). Correspondingly, the frequencies of mDCs coexpressing MitoTracker 
A

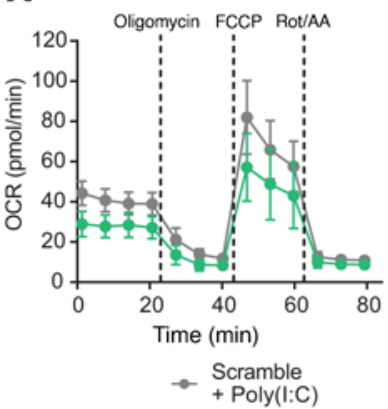

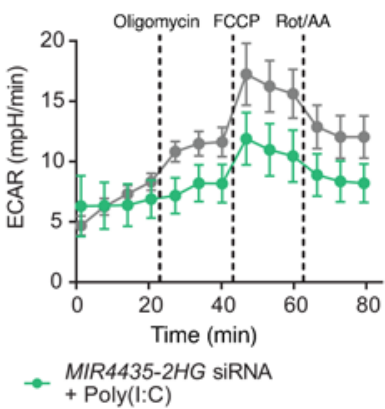

B

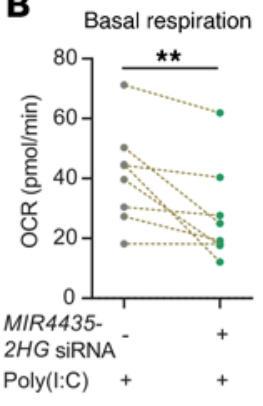

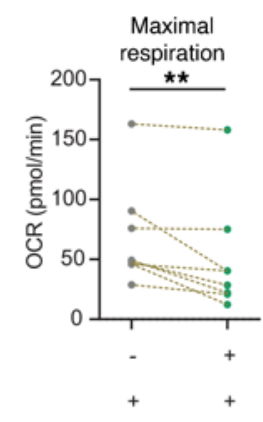

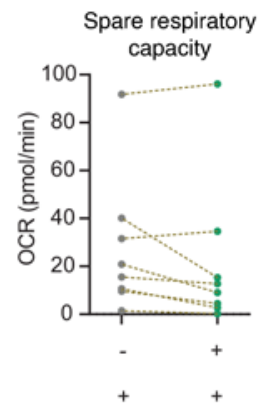

C
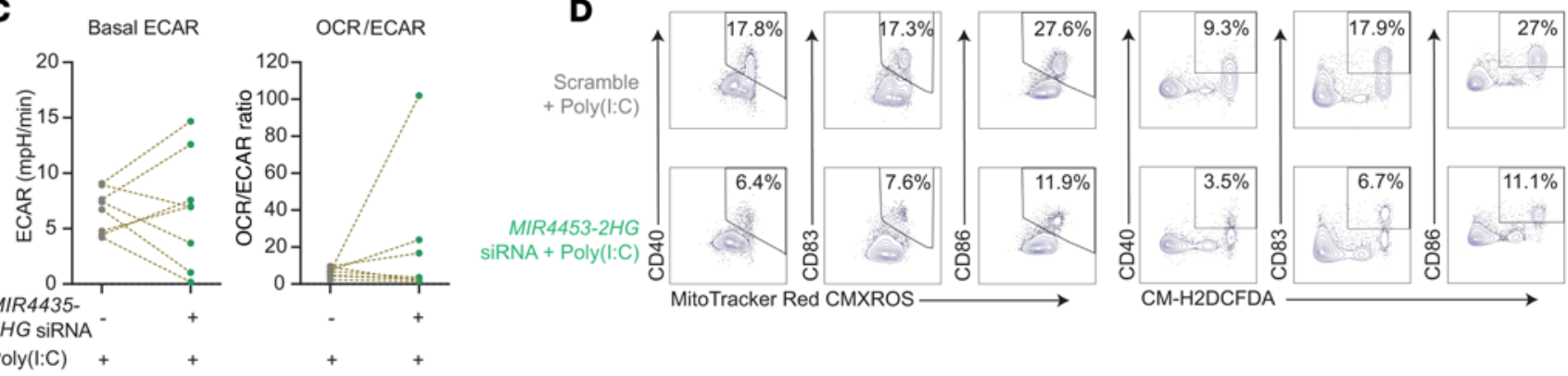

E

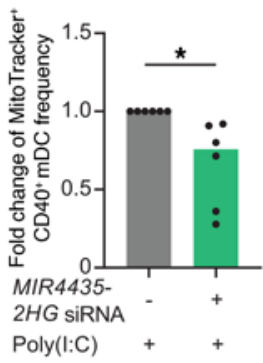

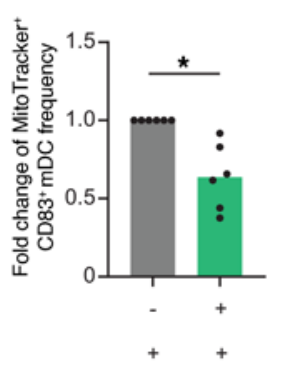

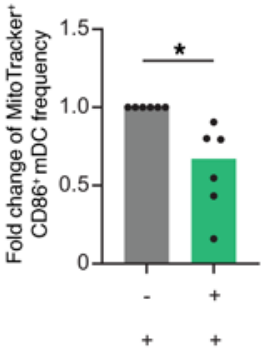

$\mathbf{F}$
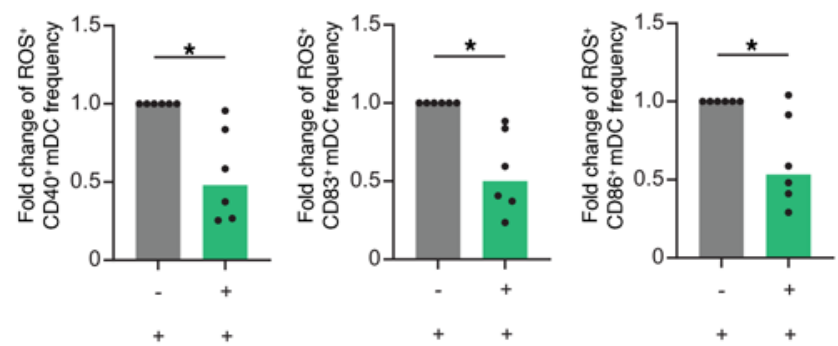

H
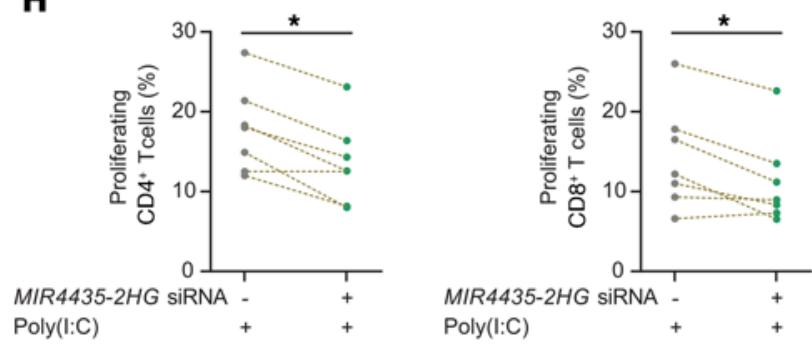

Poly(I:C)
Poly(I:C)
G

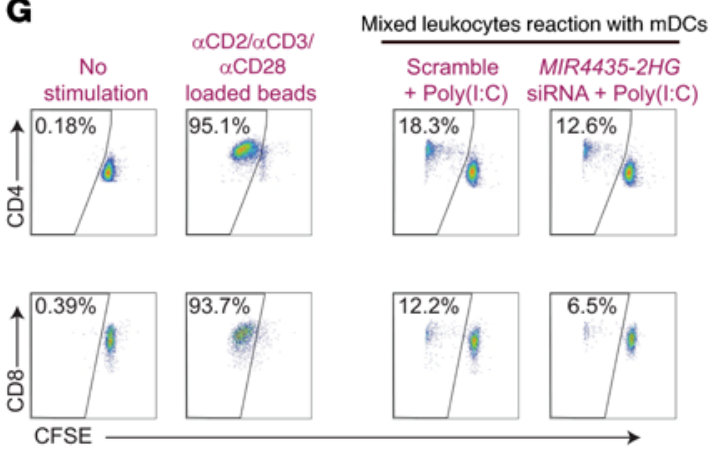

Figure 8. MIR4435-2HG modulates functional metabolic activities in mDCs. (A) OCR (left) and ECAR (right) of mDCs ( $n=8$ ) nucleofected with either MIR4435-2HG siRNA or scramble siRNA and stimulated with $2 \mu \mathrm{g} / \mathrm{mL}$ Poly(l:C) for 24 hours was measured at indicated time points in responses to oligomycin, FCCP, and rotenone/antimycin A using a Seahorse XFe96 Analyzer. (B) Basal respiration, maximal respiration, and spare respiratory capacity in mDCs from A were compared between MIR4435-2HG siRNA and scramble siRNA nucleofected cells. Wilcoxon matched pairs signed rank test was used as the statistical test. (C) Basal ECAR and OCR/ECAR ratio in mDCs from A were compared between MIR4435-2HG siRNA and scramble siRNA nucleofected cells. Wilcoxon matched pairs signed rank test was used as the statistical test. (D) Contour plots from a representative donor displaying the coexpression of MitoTracker (left) or ROS (right) to activation markers (CD40, CD83, and CD86) in mDCs nucleofected with either MIR4435-2HG siRNA or scramble siRNA and stimulated with $2 \mu \mathrm{g} / \mathrm{mL}$ Poly(l:C) for 24 hours. (E, F) The fold changes of frequencies of mDCs coexpressing MitoTracker or ROS and activation markers (CD40, CD83, and CD86) from D were compared. Wilcoxon matched pairs signed rank test was used as the statistical test. (G) A pseudocolor plot from a representative donor displaying allogeneic $C D 4^{+}$and $C D 8^{+} T$ cell proliferations following the coculture with mDCs nucleofected with MIR4435-2HG siRNA or scramble siRNA and stimulated with $2 \mu \mathrm{g} / \mathrm{mL}$ Poly(I:C). (H) The frequencies of proliferating CD4 ${ }^{+}$and $C D 8^{+} T$ cells in mixed leukocyte reactions were compared $(n=7)$. Wilcoxon matched pairs signed rank test was used as the statistical test. ${ }^{*} P<0.05,{ }^{* *} P<0.01,{ }^{* * *} P<0.001,{ }^{* * * *} P<0.0001$. 


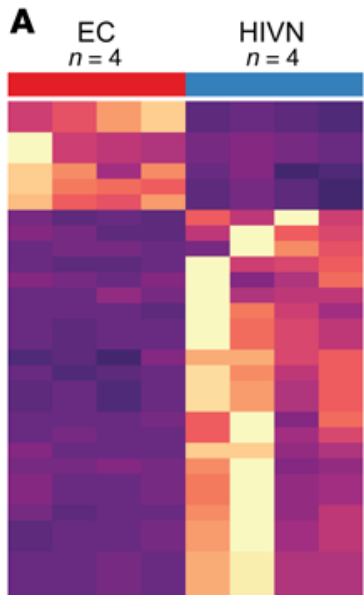

Adjusted $P$ value $<0.05$

Row z-score

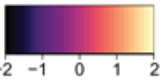

B

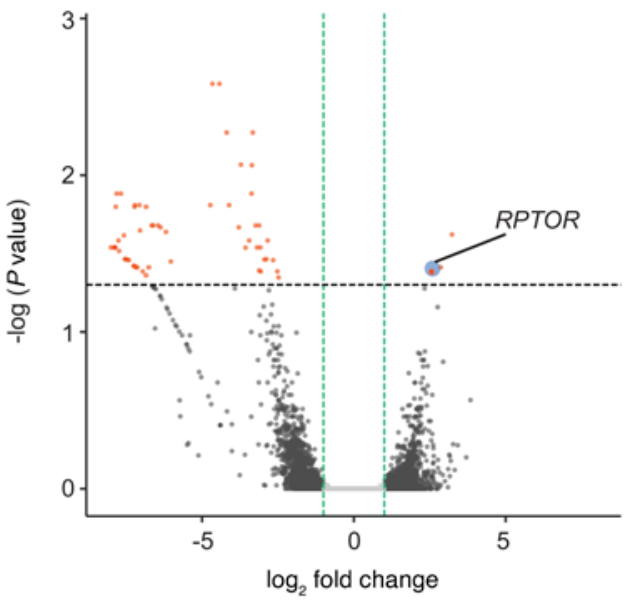

C

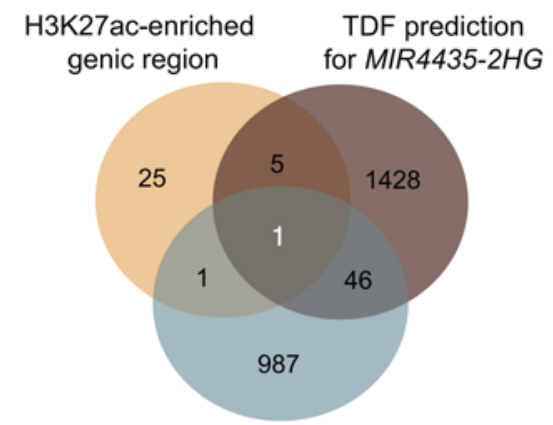

DEGs of MIR4435-2HG siRNA vs. scramble (Polyl:C-stimulated)

D $\quad \operatorname{chr} 17: 80544838-80966368$

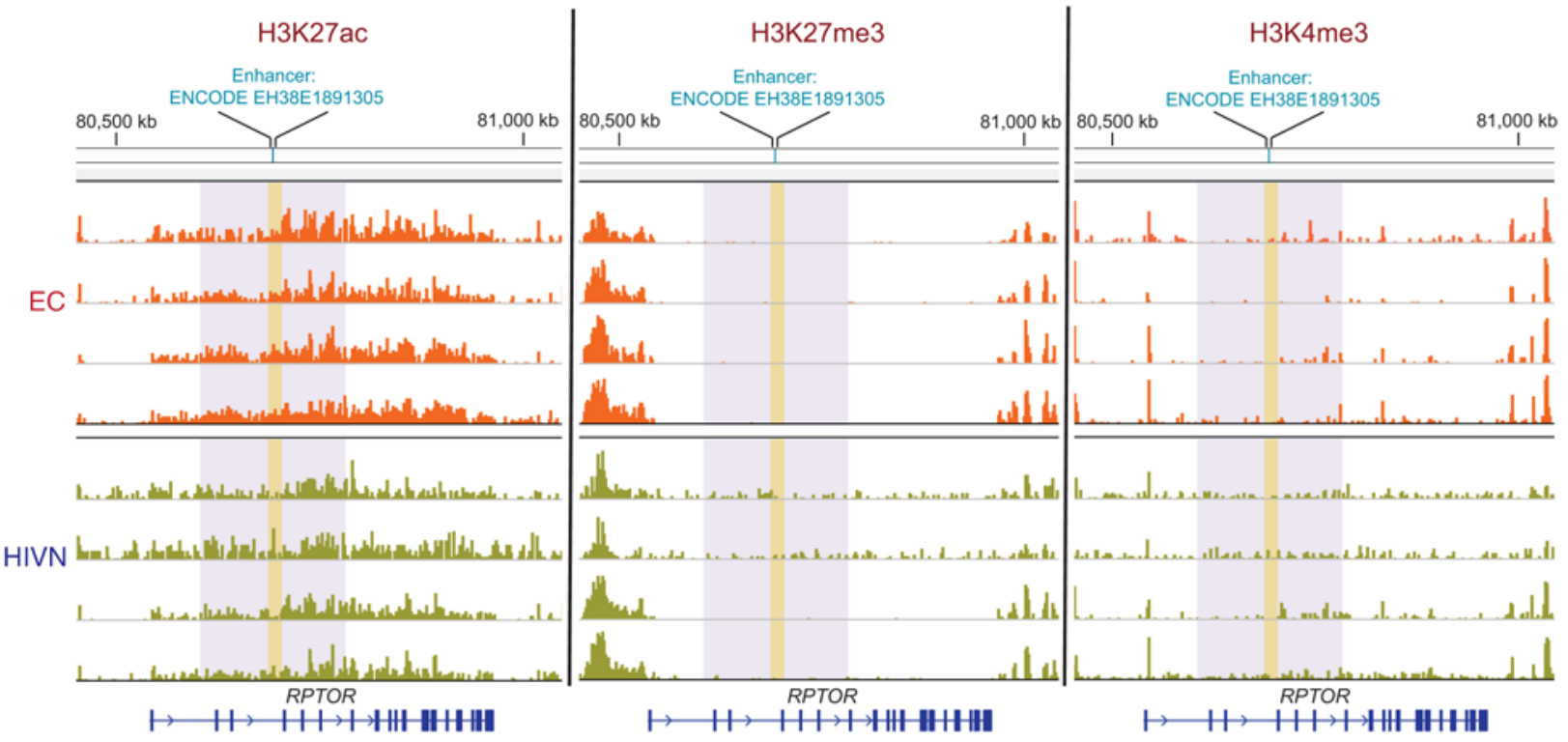

Significantly enriched region for H3K27ac Predicted MIR4435-2HG binding site by TDF

| ENCODE predicted distal enhancer (EH38E1891305)

E

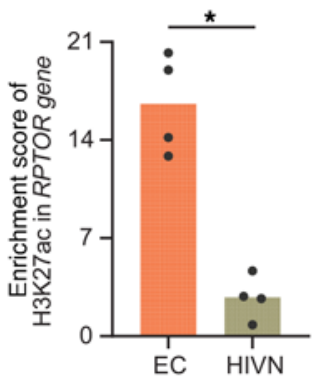

$\mathbf{F}$

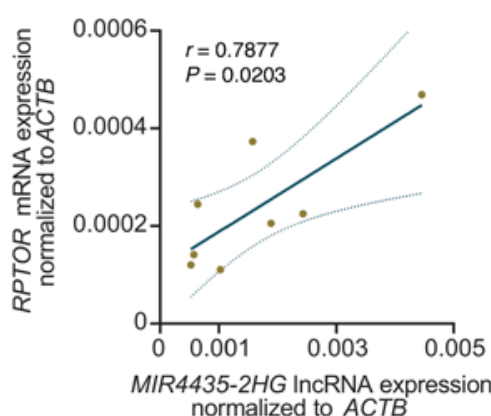

G

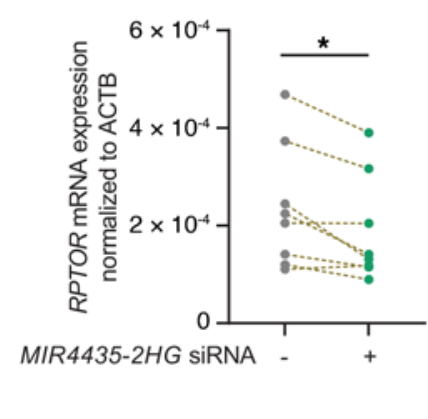

H

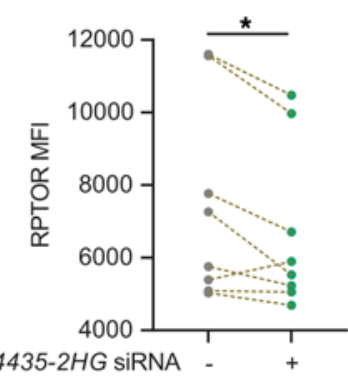


Figure 9. MIR4435-2HC influences RPTOR expression in mDCs through H3K27ac histone modification. (A) Heatmap displaying genomic loci with significant (FDR-adjusted $P$ value $<0.05)$ H3K27ac enrichment in ECs $(n=4)$ vs. HIVNs $(n=4)$. (B) Volcano plot showing H3K27ac-enriched genomic loci in ECs vs. HIVNs. Red dots represent data with $-\log (P$ value $)>1.3$ and $\log ^{2}$ fold change $>1$. (C) Venn diagram showing overlap between H3K27ac-enriched regions in $\mathrm{mDCs}$ from $\mathrm{ECs}$, genomic locations susceptible to MIR4435-2HGdependent triple-helix formation predicted by TDF, and DEGs distinguishing mDCs treated with MIR4435-2HG siRNA vs. scramble siRNA. (D) CUT\&RUNSeq reads for $\mathrm{H} 3 \mathrm{~K} 27 \mathrm{ac}, \mathrm{H} 3 \mathrm{~K} 27 \mathrm{me}$, and $\mathrm{H} 3 \mathrm{~K} 4 \mathrm{me} 3$ at the RPTOR gene locus in ECs $(n=4)$ vs. HIVNs $(n=4)$. Yellow region marks an intronic enhancer region with significantly increased $\mathrm{H} 3 \mathrm{~K} 27 \mathrm{ac}$ reads and gray region highlights the TDF-predicted MIR4435-2HG binding site. (E) The enrichment score for H3K27ac CUT\&RUN-Seq reads in the intronic enhancer region of the RPTOR gene was compared between ECs and HIVNs. Mann Whitney $U$ test was used as the statistical test. (F) Correlation analysis between MIR4435-2HG IncRNA and RPTOR mRNA expressions after normalization to ACTB in mDCs from ECs $(n=8)$. Pearson test was used to analyze the correlation. (C) The relative expression of RPTOR mRNA normalized to $A C T B$ in mDCs from ECs $(n=8)$ nucleofected with either MIR4435-2HG siRNA or scramble siRNA for 48 hours was measured by RT-PCR. Wilcoxon matched pairs signed rank test was used as the statistical test. (H) The MFI of RPTOR protein expression in mDCs from EC $(n=8)$ nucleofected with either MIR4435-2HG siRNA or scramble siRNA for 48 hours was measured by flow cytometry. Wilcoxon matched pairs signed rank test was used as the statistical test. ${ }^{*} P<0.05$, ${ }^{* *} P<0.01,{ }^{* *} P<0.001,{ }^{* * *} P<0.0001$.

or ROS with $\mathrm{CD} 40$ or $\mathrm{CD} 86$ were significantly reduced following rapamycin treatment; however, this was not seen in $\mathrm{CD} 83^{+} \mathrm{mDCs}$ coexpressing MitoTracker and ROS (Figure 10, D-G). Corresponding to these observations, the ability of rapamycin-treated mDCs to stimulate allogeneic $\mathrm{CD} 4^{+}$and $\mathrm{CD} 8^{+} \mathrm{T}$ cell proliferation was significantly lower (Figure 10, $\mathrm{H}$ and I), corresponding to our observation that mDCs exposed to MIR4435-2HG-specific siRNA had a weak ability to stimulate allogeneic T cells (Figure 8, G and H). Taken together, these experimental findings support the hypothesis that lncRNA MIR 4435-2HG can increase functional and metabolic activities of mDCs through targeted increase of mTOR function.

\section{Discussion}

A vigorous HIV-1-specific $\mathrm{T}$ cell response is commonly regarded as the backbone of antiviral immunity in persons with natural control (2), but increasing evidence suggests a contribution of innate immune cells to such a remarkable disease outcome (43-45). mDCs are of particular interest in this context, as they play key roles in priming of antiviral $\mathrm{T}$ cell responses, and seemingly have the ability to persist long-term in functionally enhanced modification states in ECs $(11,12)$, for reasons that have escaped clarification to this point. Here, we demonstrate a distinct immunometabolic profile in $\mathrm{mDCs}$ from ECs, characterized by signs of increased oxidative and nonoxidative metabolic activity. Etiologically, this altered metabolic signature was linked to overexpression of the lncRNA MIR4435-2HG, which induced increased RPTOR gene expression, likely through targeted triple-helix formation at an intronic enhancer region in the RPTOR gene and subsequent enrichment of activating histone marks at this gene locus. Together, these observations suggest that IncRNA-associated epigenetic histone modifications contribute to an enhanced metabolic and functional profile of mDCs in ECs. We propose that such an altered metabolic innate immune cell signature displays features of trained innate immunity - a concept that invokes epigenetic histone modifications as structural correlates for durable enhancements in metabolic states and functional responsiveness of innate immune cells $(13,14)$.

Understanding mechanisms enabling natural control of HIV-1 replication remains a puzzling and perplexing research question, but may possibly have widespread implications for HIV-1 cure research. Recent data underscore the relevance of immune-mediated selection mechanisms for driving a highly distinct viral reservoir configuration in these individuals, characterized by chromosomal locations of intact proviruses that display features of deep latency (4). While $\mathrm{T}$ cell-mediated responses, specifically when targeting highly networked viral epitopes, may play a key role in these selection processes (46), a contribution of innate immune cells appears increasingly obvious, as marked variations in quantity and quality have been reported for NK cells $(47,48)$, pDCs (49-51), and, most frequently, for mDCs from controllers (11, 12, 52, 53). Here, we extend these findings by showing a distinct expression pattern of OXPHOS and mTOR genes in mDCs from ECs, which was coupled with an enhanced oxidative and nonoxidative metabolic profile in response to TLR3 stimulation. Notably, this altered functional metabolic state in ECs was associated with selective upregulation of MIR4435-2HG, a lncRNA that we show can directly influence the metabolic state of $\mathrm{mDCs}$ through induction of RPTOR expression and ensuing increases in mTORC1 activity. Importantly, a distinct MIR4435-2HG-related immunometabolic profile occurred selectively in mDCs from ECs, but was absent in HAARTs and CPs, suggesting that it may represent an intrinsic component of antiviral immune defense by ECs and not a mere consequence of virological control (52).

A contribution of lncRNAs to regulation of pathogen-specific immune responses has been documented in a variety of different contexts (17), and is frequently mediated through direct interactions with host transcription factors influencing the expression of immune-related genes $(13,14)$. Direct protein-RNA interactions between IncRNA MIR4435-2HG and protein components of the host transcriptional machinery have indeed been observed in specific cell types $(54,55)$, and can therefore not be fully excluded as underlying explanations of our observations. A more likely mechanism, however, involves IncRNA-DNA interactions that are facilitated by triple-helix formation. Triple-helix structures between lncRNAs and double-stranded chromosomal DNA are experimentally well documented, and can occur when lncRNAs are forming Hoogsteen or reverse Hoogsteen hydrogen bonds with purinerich DNA (40). Notably, only specific base combinations within defined segments of IncRNA and chromosomal DNA can promote such complex molecular structures (40). In the case of MIR4435$2 H G$, computational algorithms predicted triple-helix RNA-DNA conformations at an intronic enhancer region of the RPTOR gene, exactly at a location where epigenetic testing revealed enhanced activating H3K27ac signals in mDCs from ECs. Increased recruitment of histone acetyltransferases is indeed a recognized effect of triple-helix lncRNA-DNA interactions (39). While structural biology experiments would be necessary to formally demonstrate a triple-helix configuration with MIR4435-2HG at the RPTOR gene locus, we propose that the combined experimental and computational results make such lncRNA-DNA interaction highly likely. Notably, synergistic effects among lncRNAs in the regulation of 
A

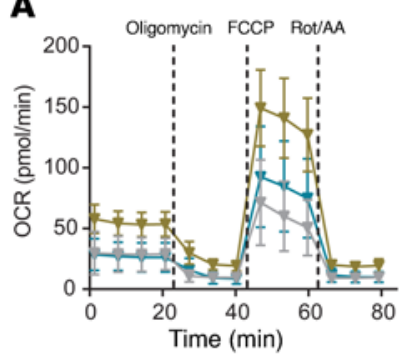

* Medium $\quad$ - Poly $(\mathrm{l}: \mathrm{C})$

C

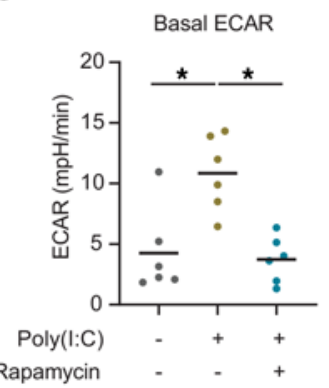

D

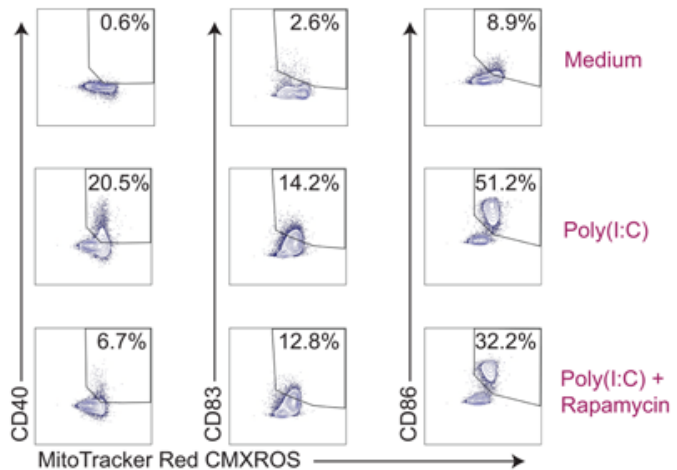

E

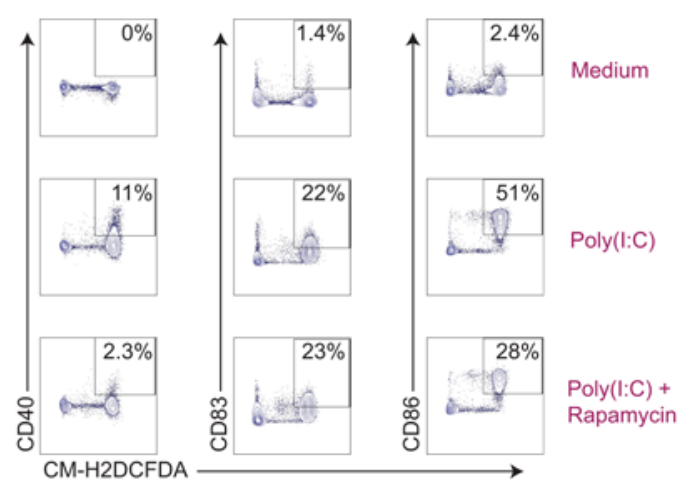

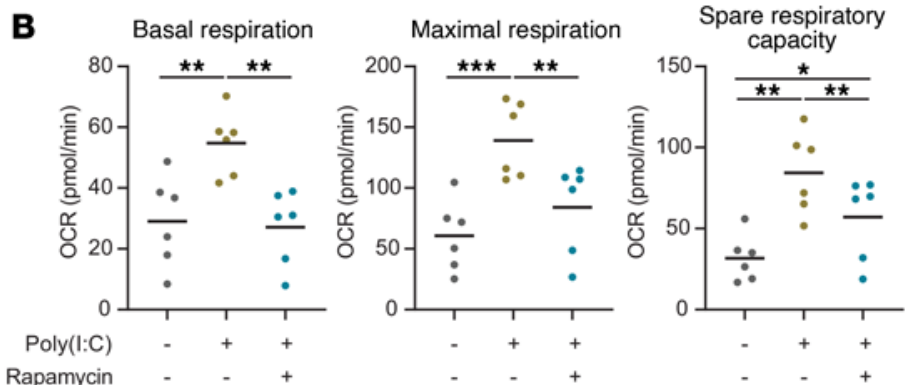

$\mathbf{F}$

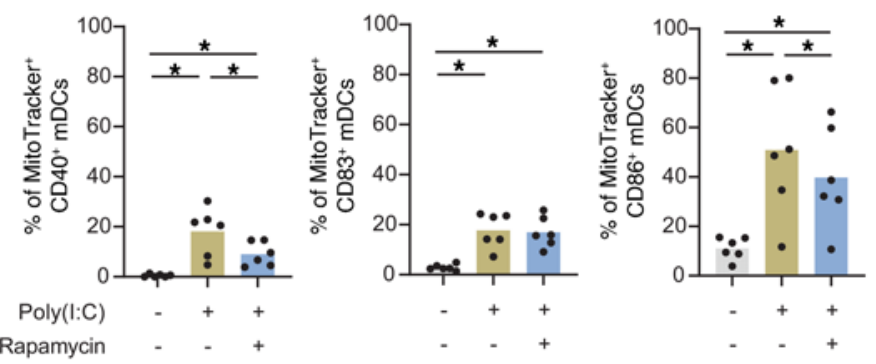

G
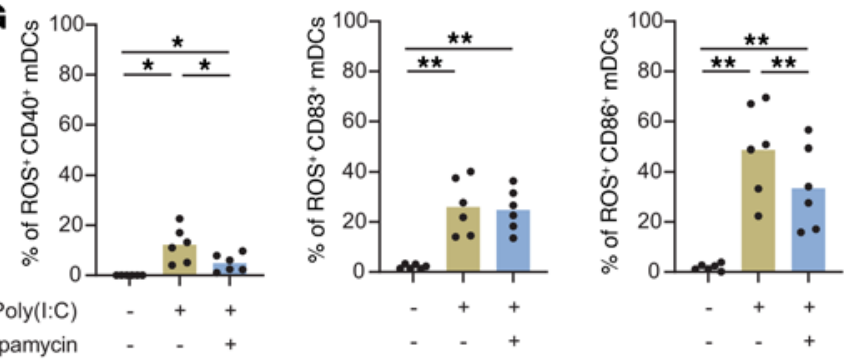

Rapamyci

$\mathbf{H}$

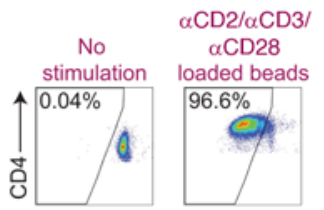

Mixed leukocytes reaction
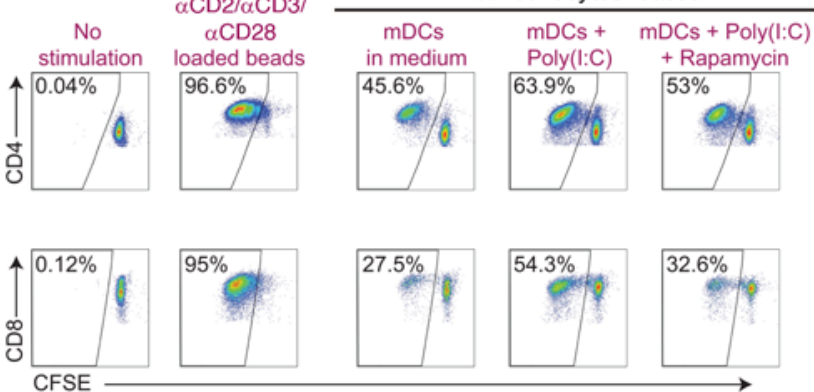

$32.6 \%$

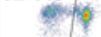

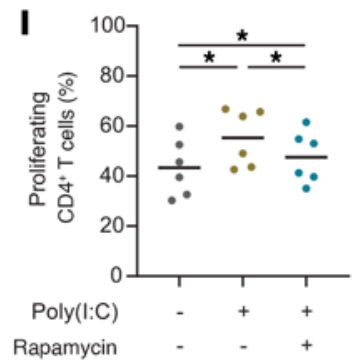

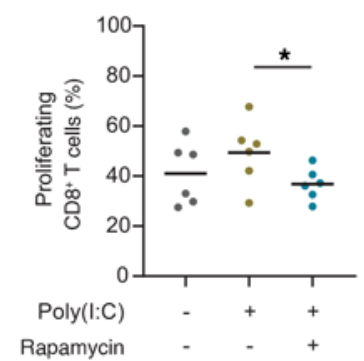


Figure 10. mTORC1 inhibition reduced OXPHOS, glycolysis, and functional activities in mDCs. (A) OCR (left) and ECAR (right) of mDCs from HIVNs ( $n=$ 6) stimulated in $2 \mu \mathrm{g} / \mathrm{mL}$ Poly $(\mathrm{I}: \mathrm{C})$ with or without $100 \mathrm{nM}$ rapamycin for 24 hours were measured at indicated time points in responses to oligomycin, FCCP, and rotenone/antimycin A using a Seahorse XFe96 Analyzer. mDCs cultured in medium only served as a negative control. (B) Basal respiration, maximal respiration, and spare respiratory capacity in $\mathrm{mDC}$ s from $\mathbf{A}$ were compared in mDCs stimulated with $2 \mu \mathrm{g} / \mathrm{mL}$ Poly(l:C), with or without 100 $\mathrm{nM}$ rapamycin for 24 hours. Repeated measures 1-way ANOVA test was used for statistical analysis. (C) Basal ECAR and OCR/ECAR ratio in mDCs from $\mathbf{A}$ were compared in $\mathrm{mDCs}$ stimulated with $2 \mu \mathrm{g} / \mathrm{mL}$ Poly $(\mathrm{I}: \mathrm{C})$, with or without $100 \mathrm{nM}$ rapamycin for 24 hours. Repeated measures 1-way ANOVA test was used for statistical analysis. (D, E) Contour plots from a representative donor displaying the coexpression of MitoTracker or ROS and activation markers (CD40, CD83, and CD86) in mDCs stimulated with $2 \mu \mathrm{g} / \mathrm{mL}$ Poly(l:C), with or without $100 \mathrm{nM}$ rapamycin for 24 hours. $(\mathbf{F}, \mathbf{C})$ The frequencies of mDCs coexpressing MitoTracker or ROS and activation markers (CD40, CD83, and CD86) in mDCs from HIVNs $(n=6)$ in $\mathbf{D}$ and $\mathbf{E}$ were compared. Repeated measures 1-way ANOVA test was used for statistical analysis. (H) A pseudocolor plot from a representative donor displaying allogeneic $C D 4^{+}$and $C D 8^{+}$ T cell proliferations following the coculture with $\mathrm{mDCs}$ stimulated with 2 $\mu \mathrm{g} / \mathrm{mL}$ Poly(I:C), with or without $100 \mathrm{nM}$ rapamycin for 24 hours. (I) The frequencies of proliferating $\mathrm{CD} 4^{+}$and $\mathrm{CD} 8^{+} \mathrm{T}$ cells in mixed leukocyte reactions were compared $(n=6)$. Repeated measures 1-way ANOVA test was used for statistical analysis. ${ }^{*} P<0.05,{ }^{* *} P<0.01,{ }^{* * *} P<0.001,{ }^{* * *} P<0.0001$

immune cell functions are yet to be explored, as our data have shown that additional lncRNAs were specifically upregulated in mDCs from ECs. Therefore, future studies may be dedicated to investigating these additional lncRNAs and their possible combinatorial roles in regulating immune cell behaviors.

The importance of metabolic profiles for dictating immune cell function is well recognized in ECs and in other conditions (56-58). Resting naive and memory $\mathrm{T}$ cells use mitochondrial OXPHOS for a steady supply of energy (59), which upon activation shifts toward glycolysis and elevated mitochondrial biogenesis in order to meet higher energy demands $(56,57,59)$. A similar immunometabolic process can be seen in innate immune cells such as DCs and macrophages, which typically rely on the tricarboxylic acid (TCA) cycle and on OXPHOS as the main energy source during resting conditions $(60,61)$. Once activated, nonoxidative energy production via glycolysis can dominate (61-63). In our study, we observed higher OXPHOS and glycolysis in mDCs from ECs after stimulation with the TLR3 ligand Poly(I:C), without affecting the OCR/ECAR ratio. This corresponded to the observation that both MIR4435-2HG gene silencing and pharmacological inhibition of mTORC1 with rapamycin decreased oxidative and nonoxidative metabolic activities in mDCs. Collectively, these data strongly suggest a role for the MIR4435-2HG/RPTOR axis in enhancing the metabolic activity of mDCs through upregulation of mTORC1 function. While such an enhanced metabolic state is likely to increase the functional responsiveness of $\mathrm{mDCs}$, in particular with regard to costimulatory activities, it is noteworthy that continuous MIR4435-2HG-associated increases in oxidative phosphorylation may be associated with a risk for elevated oxidative damage resulting from ROS. Therefore, it is possible that an elevated functional and metabolic profile in $\mathrm{mDC}$ s from ECs is achieved at the expense of elevated detrimental oxidative stress.

Durable persistence of innate immune cells in higher functional modification states is well conceptualized in the context of trained immunity, and frequently involves epigenetic modifications and remodeling of chromatin configurations $(14,64-66)$. For instance, epigenetic modifications induced by the Bacillus Calmette-Guérin (BCG) vaccine can lead to enrichment of H3K27ac marks at active promoters and enhancers, and lead to improved functional responsiveness to antimicrobial antigen challenges (67). Such a BCG-associated training of innate immunity may translate into health benefits in a variety of disease contexts $(68,69)$. Our data raise the hypothesis that in addition to highly effective $\mathrm{T}$ cell immune responses, ECs may be characterized by a functionally enhanced immune profile in $\mathrm{mDCs}$ that is related to distinct epigenetic chromatin features and may result from prior exposure to HIV-1 and/or additional microbial antigens. Future studies will be necessary to determine whether epigenetically programmed features of improved innate immune functions in ECs might translate into a more effective immune activity against pathogens other than HIV-1. Moreover, profiling of the human epigenome in defined innate immune cells from ECs will be helpful in characterizing possible additional components of trained immunity in ECs and may represent a complementary effort to large-scale genomic studies conducted previously in this particular population (70). We remain optimistic that continuous progress in defining the diverse components of protective immunity in ECs may ultimately allow the induction of spontaneous control of HIV-1 in larger populations of persons living with HIV-1.

\section{Methods}

Study participants. HIV-1 ECs $(n=23)$ who had maintained undetectable levels of HIV-1 replication for a median of 5 years in the absence of antiretroviral therapy (viral load $<50$ copies $/ \mathrm{mL}$; median $\mathrm{CD}_{4}{ }^{+} \mathrm{T}$ cell count 882 cells $/ \mathrm{mm}^{3}$ ), HAARTs $(n=13$; viral load $<50$ copies $/ \mathrm{mL}$; median $\mathrm{CD}^{+} \mathrm{T}$ cell count 909 cells $\left./ \mathrm{mm}^{3}\right)$, CPs $(n=9$; median viral load 53,500 copies $/ \mathrm{mL}$; median $\mathrm{CD} 4^{+} \mathrm{T}$ cell count 528 cells $\left./ \mathrm{mm}^{3}\right)$, and HIVNs $(n=15)$ were recruited for this study. The clinical and demographic characteristics of the study subjects are listed in Supplemental Table 1. Additional PBMC samples from HIV-1-negative healthy donors at the MGH Blood Bank were obtained for gene knockdown and mixed leukocyte reaction experiments.

Magnetic isolation of $m D C s . \mathrm{mDCs}$ from peripheral blood were isolated using Human Myeloid Dendritic Cells Isolation Kit (Miltenyi) and LD columns by negative selection. The purity of cells after isolation was greater than $90 \%$. The isolated $\mathrm{mDCs}$ were used for downstream assays.

Gene expression analysis by RNASeq. Total RNA from isolated mDCs was extracted and purified using the PicoPure RNA Isolation Kit (Applied Biosystems). Subsequently, RNAseq libraries were generated as previously described (71). The whole transcriptome amplification (WTA) and tagmentation-based library preparation were performed using Nextera XT (Illumina), followed by sequencing on a NextSeq 500 Instrument (Illumina). Sequences from RNAseq were aligned to the human genome (GRCh38) using STAR (72) and quantified using RSEM (73). Raw counts at gene or isoform levels were normalized using External RNA Controls Consortium spikedin controls through RUV-seq (74), and then used for differential gene expression analysis with DESeq2 (75). Transcripts per million (TPM) values were used for downstream analysis.

Differentially expressed genes (DEGs) from previously generated RNAseq data set (Gene Expression Omnibus accession GSE141498) (25) and current RNAseq data set (GSE167565 and GSE167564) were 
analyzed using the IPA (Qiagen) (28) and the Database for Annotation, Visualization, and Integrated Discovery (DAVID) v6.8 (29, 30).

In vitro cell culture. Isolated $\mathrm{mDCs}$ or total PBMCs were cultured in medium only (negative control) or with $2 \mu \mathrm{g} / \mathrm{mL}$ TLR3 ligand Poly(I:C) (Invivogen) for 24 hours. The cells were cultured in RPMI medium (Thermo Fisher Scientific) supplemented with 10\% fetal bovine serum (FBS) (MilliporeSigma), 1\% L-glutamine (Corning), 1\% penicillin/streptomycin (Corning), and 1\% HEPES buffer (Corning) and kept at $37^{\circ} \mathrm{C}$ in $5 \% \mathrm{CO}_{2}$ during the culture. In some experiments, isolated $\mathrm{mDCs}$ were stimulated in $2 \mu \mathrm{g} / \mathrm{mL}$ TLR3 ligand Poly(I:C) (Invivogen), with or without $100 \mathrm{nM}$ rapamycin (Invivogen) for 24 hours.

Oxygen consumption rate measurement. The OCR was analyzed using the Seahorse Mito Stress Test Kit (Agilent) on a Seahorse XFe96 Analyzer (Agilent) according to the manufacturer's protocol. Briefly, conditioned mDCs were harvested, washed using prewarmed supplemented RPMI medium, and plated on XF96 cell culture microplates (Agilent) coated with poly-D-lysine (Thermo Fisher Scientific) to adhere the cells on the microplate surface. Prior to the assay, the RPMI medium was replaced with XF Base medium with minimal DMEM (Agilent), supplemented with $1 \mathrm{mM}$ pyruvate (Agilent), $2 \mathrm{mM}$ glutamine (Agilent), and $10 \mathrm{mM}$ glucose (Agilent) with the $\mathrm{pH}$ adjusted to 7.4. At indicated time points, OCR was measured in the basal condition and in response to $2 \mu \mathrm{M}$ oligomycin, $3 \mu \mathrm{M}$ FCCP (carbonyl cyanide- 4 (trifluoromethoxy) phenylhydrazone), and $1 \mu \mathrm{M}$ rotenone/antimycin A (Agilent).

Mitochondrial potential and ROS measurement. Mitochondrial membrane potential and ROS were measured by staining cells using $100 \mathrm{nM}$ MitoTracker Red CMXROS (Invitrogen) and $5 \mu \mathrm{M}$ CM-H2DCFDA (Invitrogen) probes, respectively. Total PBMCs or isolated $\mathrm{mDCs}$ were incubated for 30 minutes in these probes at $37^{\circ} \mathrm{C}$. Cells were then washed and stained with LIVE/DEAD Blue Viability Dye (Invitrogen) and fluorophore-conjugated monoclonal antibodies targeting CD3, CD14, CD16, CD19, CD20, CD56, HLA-DR (BD Biosciences), CD11c, CD40, CD83, and CD86 (Biolegend). The expression of mitochondrial membrane potential and ROS was measured by a BD Symphony instrument (BD Biosciences). The data were analyzed using FlowJo software (FlowJo, LLC).

RT-PCR. mDCs were washed in $1 \times$ PBS and lysed using PicoPure Extraction Buffer (Applied Biosystems). Total RNA was extracted and purified using the PicoPure RNA Isolation Kit (Applied Biosystems), according to the manufacturer's protocol. Next, total RNA was reverse transcribed using SuperScript IV Reverse Transcriptase (Invitrogen) into cDNA. Quantitative PCR was performed using the Quantitect SYBR Green PCR Kit (Qiagen) and primers designed to amplify MIR44352HG. A ViiA7 instrument (Life Technologies) was used. ACTB was analyzed as a housekeeping gene. The primer sequences are displayed in Supplemental Table 3. The expression of MIR4435-2HG relative to the negative control was calculated using the Livak $\left(2^{(-\Delta \Delta C t)}\right)$ method.

PrimeFlow RNA FACS for lncRNA. The expression of lncRNA MIR4435-2HG in relation to various DC activation markers in different DC subsets were evaluated using a flow cytometry-based PrimeFlow RNA assay kit (Invitrogen). Initially, total PBMCs (5 million cells) were stained using LIVE/DEAD Blue Viability Dye (Invitrogen) and fluorophore-conjugated monoclonal antibodies specific for various surface markers (Supplemental Table 4). The cells were then fixed and permeabilized using the PrimeFlow fixation and permeabilization kit according to the manufacturer's protocol. Subsequently, a MIR4435-2HG-targeting probe, PreAmp, Amp, and label probe hybridizations were performed at $40^{\circ} \mathrm{C}$ at indicated durations of incubation. Specific probes targeting the housekeeping gene ACTB served as a positive control, while specific target probes were omitted in negative controls. The expression of lncRNA MIR4435-2HG and other cell markers was measured using a BD Symphony instrument (BD Biosciences). Data were analyzed using FlowJo software (FlowJo, LLC) with plugins for tSNE and FlowSOM (34). TSNE analysis was performed in 24,336 cells using equal sampling of cell numbers between ECs and HIVNs, with 1000 iterations, a perplexity of 5, and learning rate (eta) of 1703.

siRNA-mediated lncRNA knockdown. Knockdown of the lncRNA MIR4435-2HG was performed by nucleofecting isolated primary mDCs (program CM120, Amaxa 4D-Nucleofector, Lonza) with specific or scramble siRNAs (Horizon Dharmacon) according to the manufacturer's instructions. Nucleofected mDCs were cultured in RPMI medium (Thermo Fisher Scientific) without antibiotics and supplemented with 10\% FBS (MilliporeSigma), 1\% L-glutamine (Corning), and 1\% HEPES buffer (Corning) for 24 or 48 hours. Subsequently, the cells were either stimulated with $2 \mu \mathrm{g} / \mathrm{mL}$ TLR3 ligand Poly(I:C) (Invivogen), $100 \mathrm{ng} / \mathrm{mL}$ TLR4 ligand LPS (Invivogen), $5 \mu \mathrm{g} / \mathrm{mL}$ TLR7/8 ligand CL097 (Invivogen), or not given any stimulation (medium only as a negative control) for another 24 hours. Efficiency of siRNA-mediated knockdown was confirmed at the RNA level by RT-PCR.

CUT\&RUN sequencing. A standard CUT\&RUN sequencing protocol was used (41) with minor modifications. Briefly, isolated mDCs from ECs and HIVNs were bound to concanavalin A-coated magnetic beads (Bangs Laboratories), followed by cell permeabilization using $0.01 \%$ digitonin (Millipore) in wash buffer. Primary antibodies against intracellular H3K27ac, H3K27me3, and H3K4me3 histone marks (Cell Signaling Technology) were added and cells were incubated at $4^{\circ} \mathrm{C}$ for overnight. Guinea pig anti-rabbit IgG antibody (Antibodies-online) was used as a negative control. The next day, fusion protein CUTANA pA/G-MNase (20x) (Epicypher) was added and cells were incubated at $4^{\circ} \mathrm{C}$ for 1 hour, followed by chromatin digestion using $100 \mathrm{mM} \mathrm{CaCl}_{2}$ (Thermo Fisher Scientific). Stop buffer was then added to stop the chromatin digestion. DNA fragments were extracted using phenol chloroform (Invitrogen) and the extracted DNA was eluted in $1 \mathrm{mM}$ Tris- $\mathrm{HCl} \mathrm{pH}$ 8.0 (Thermo Fisher Scientific) + 0.1 mM EDTA (Sigma-Aldrich). The DNA library was prepared using NEBNext Ultra II DNA Library Prep Kit for Illumina Sequencing (New England Biolabs). The DNA library concentration and quality were measured by Qubit $1 \times$ dsDNA HS kit (Life Technologies) and D1000 High Sensitivity TapeStation (Agilent), respectively. The sequencing was performed using NextSeq 500/550 High Output v2.5 kit (75 cycles) (Illumina) on a NextSeq 500 Instrument (Illumina). Adapters and low-quality reads were trimmed using Trimmomatic (76) and aligned to the human genome (GRCh38) using Bowtie2 (77). Peak calling was implemented using MACS2 (78). For visualization, the coverage profile was calculated using DeepTools (79). Differential binding analysis was performed using DiffBind (80). The CUT\&RUN sequencing data are available in Gene Expression Omnibus accession GSE167563.

TDF analysis for IncRNA. The binding sites between candidate lncRNA and DNA were characterized using TDF (40). The software was first used to computationally predict the potential binding sites for our candidate lncRNA. The promoter regions of genes from multiple pathways were then tested statistically to identify regions enriched with lncRNA binding sites.

Mixed leukocyte reaction. Total allogeneic T cells were isolated using Dynabeads Untouched Human T Cells Kit (Invitrogen) and rested over- 
night at $37^{\circ} \mathrm{C}$ in $5 \% \mathrm{CO}_{2}$. The next day, isolated $\mathrm{T}$ cells were stained using the Vybrant CFDA SE Cell Tracer Kit (Invitrogen) and washed in complete RPMI medium. Total T cells were then cocultured with conditioned $\mathrm{mDC}$ for 5 days at a $\mathrm{T} / \mathrm{mDC}$ ratio of $4: 1$. The cells were cultured in RPMI medium (Thermo Fisher Scientific) supplemented with 10\% FBS (MilliporeSigma), 1\% L-glutamine (Corning), 1\% penicillin/streptomycin (Corning), and $1 \%$ HEPES buffer (Corning) and kept at $37^{\circ} \mathrm{C}$ in $5 \% \mathrm{CO}_{2}$ during the culture. As a negative control, total $\mathrm{T}$ cells were cultured in medium only, and as a positive control, $\mathrm{T}$ cells were cultured with $\mathrm{T}$ cell activation beads loaded with $\alpha \mathrm{CD} 2 / \alpha \mathrm{CD} 3 / \alpha \mathrm{CD} 28$ antibodies (Miltenyi).

Statistics. Differences between 2 groups were tested for statistical significance using unpaired, 2-tailed $t$ test or Mann Whitney test (unpaired observations) and paired, 2-tailed $t$ test or Wilcoxon matched pairs signed rank test (paired observations). Differences among 3 groups or more were tested using 1-way ANOVA or Kruskal-Wallis (unpaired observations) and repeated measures ANOVA or Friedman test (paired observations). Correlation between 2 variables was tested using Spearman or Pearson test. Normality distribution was tested using Kolmogorov Smirnov test.

Study approval. All subjects gave written informed consent and the study was approved by the IRB of Massachusetts General Hospital.

\section{Author contributions}

XGY and ML conceptualized the study. XGY, ML, and CAH determined the study methodology. CG performed software and computational analyses. CAH, YR, and EMG conducted the study investigation. CAH and CG conducted the formal study analysis. $\mathrm{XGY}, \mathrm{ML}$, and BDW contributed resources. XGY, ML, and CAH wrote the original draft of the manuscript. YR and EMG reviewed and edited the manuscript. XGY and ML supervised the study. XGY and ML acquired study funding.

\section{Acknowledgments}

XGY is supported by NIH grants HL134539, AI116228, AI078799, DA047034, AI155171, and AI150396 and by the Bill and Melinda Gates Foundation (INV-002703). ML is supported by NIH grants AI098487, AI135940, AI114235, AI117841, AI120008, AI152979, and DK120387. ML and XGY are associated members of the BEAT-HIV Martin Delaney Collaboratory (UM1 AI126620). The International HIV Controller Cohort is supported by the Bill and Melinda Gates Foundation (OPP1066973), the Ragon Institute of MGH, MIT and Harvard, the NIH (R37 AI067073 to BDW), and the Mark and Lisa Schwartz Family Foundation. We thank Facundo Batista and Johan Arnold of Ragon Institute of MGH, MIT and Harvard for facilitating the use of the Seahorse XFe96 Analyzer instrument. We also thank the Ragon Institute Flow Core Facility for assistance with flow cytometry assays.

Address correspondence to: Xu Yu, Associate Professor of Medicine, Ragon Institute of MGH, MIT, and Harvard, 400 Technology Square, Cambridge, Massachusetts 02139, USA. Phone: 857.891.8145; Email: xyu@mgh.harvard.edu.
1. Eisinger RW, et al. HIV viral load and transmissibility of HIV infection: undetectable equals untransmittable. JAMA. 2019;321(5):451-452.

2. Collins DR, et al. CD8 ${ }^{+} \mathrm{T}$ cells in HIV control, cure and prevention. Nat Rev Immunol. 2020;20(8):471-482.

3. Gurdasani D, et al. A systematic review of definitions of extreme phenotypes of HIV control and progression. AIDS. 2014;28(2):149-162.

4. Jiang $C$, et al. Distinct viral reservoirs in individuals with spontaneous control of HIV-1. Nature. 2020;585(7824):261-267.

5. Baker BM,et al. Elite control of HIV infection: implications for vaccine design. Expert Opin Biol Ther. 2009;9(1):55-69.

6. Piguet V, Steinman RM. The interaction of HIV with dendritic cells: outcomes and pathways. Trends Immunol. 2007;28(11):503-510.

7. Wu L, KewalRamani VN. Dendritic-cell interactions with HIV: infection and viral dissemination. Nat Rev Immunol. 2006;6(11):859-868.

8. Gringhuis SI, et al. HIV-1 exploits innate signaling by TLR8 and DC-SIGN for productive infection of dendritic cells. Nat Immunol. 2010;11(5):419-426.

9. Silvin A, et al. Constitutive resistance to viral infection in human CD141 ${ }^{+}$dendritic cells. Sci Immunol. 2017;2(13):eaai8071.

10. Coindre S, et al. Mass cytometry analysis reveals complex cell-state modifications of blood myeloid cells during HIV infection. Front Immunol. 2019;10:2677.

11. Martin-Gayo E, et al. Potent cell-intrinsic immune responses in dendritic cells facilitate HIV-1-specific T cell immunity in HIV-1 elite controllers. PLoS Pathog. 2015;11(6):e1004930.
12. Martin-Gayo E, et al. A reproducibility-based computational framework identifies an inducible, enhanced antiviral state in dendritic cells from HIV-1 elite controllers. Genome Biol. 2018;19(1):10.

13. Netea MG, et al. Trained immunity: a program of innate immune memory in health and disease. Science. 2016;352(6284):aaf1098.

14. Netea MG, et al. Defining trained immunity and its role in health and disease. Nat Rev Immunol. 2020;20(6):375-388.

15. Fok ET, et al. The lncRNA connection between cellular metabolism and epigenetics in trained immunity. Front Immunol. 2018;9:3184.

16. Ørom U, Shiekhattar R. Long noncoding RNAs usher in a new era in the biology of enhancers. Cell. 2013;154(6):1190-1193.

17. Yao R-W, et al. Cellular functions of long noncoding RNAs. Nat Cell Biol. 2019;21(5):542-551.

18. Marchese FP, et al. The multidimensional mechanisms of long noncoding RNA function. Genome Biol. 2017;18(1):206.

19. Wang KC, et al. A long noncoding RNA maintains active chromatin to coordinate homeotic gene expression. Nature. 2011;472(7341):120-124.

20. Wurm AA, Pina C. Long non-coding RNAs as functional and structural chromatin modulators in acute myeloid leukemia. Front Oncol. 2019;9:899.

21. Hirose T, et al. NEAT1 long noncoding RNA regulates transcription via protein sequestration within subnuclear bodies. Mol Biol Cell. 2014;25(1):169-183.

22. Imamura $\mathrm{K}$, et al. Long noncoding RNA NEAT1dependent SFPQ relocation from promoter region to paraspeckle mediates IL8 expression upon immune stimuli. Mol Cell. 2014;53(3):393-406.

23. Fanucchi $S$, et al. Immune genes are primed for robust transcription by proximal long noncoding RNAs located in nuclear compartments. Nat Genet. 2019;51(1):138-150.

24. Fanucchi S, Mhlanga MM. Lnc-ing trained immunity to chromatin architecture. Front Cell Dev Biol. 2019;7:2.

25. Martin-Gayo E, et al. Immunological fingerprints of controllers developing neutralizing HIV-1 antibodies. Cell Rep. 2020;30(4):984-996.

26. Kotzin JJ, et al. The long noncoding RNA Morrbid regulates CD8 T cells in response to viral infection. Proc Natl Acad Sci U S A. 2019;116(24):11916-11925.

27. Kotzin JJ, et al. The long non-coding RNA Morrbid regulates Bim and short-lived myeloid cell lifespan. Nature. 2016;537(7619):239-243.

28. Krämer A, et al. Causal analysis approaches in Ingenuity Pathway Analysis. Bioinformatics. 2014;30(4):523-530.

29. Huang DW, et al. Systematic and integrative analysis of large gene lists using DAVID bioinformatics resources. Nat Protoc. 2009;4(1):44-57.

30. Huang DW, et al. Bioinformatics enrichment tools: paths toward the comprehensive functional analysis of large gene lists. Nucleic Acids Res. 2009;37(1):1-13.

31. Harvey RF, et al. Signaling from mTOR to eIF $2 \alpha$ mediates cell migration in response to the chemotherapeutic doxorubicin. Sci Signal. 2019;12(612): eaaw6763.

32. Soliman GA, Schooling MC. Causal association between mTOR-dependent EIF-4E and EIF-4A circulating protein levels and type 2 diabetes: 
a Mendelian randomization study. Sci Rep. 2020;10(1):15737.

33. Previte DM, et al. Lymphocyte activation gene-3 maintains mitochondrial and metabolic quiescence in naive CD4 ${ }^{+}$T cells. Cell Rep. 2019;27(1):129-141.

34. Gassen S, et al. FlowSOM: using self-organizing maps for visualization and interpretation of cytometry data. Cytometry A. 2015;87(7):636-645.

35. Villani A-CC, et al. Single-cell RNA-seq reveals new types of human blood dendritic cells, monocytes, and progenitors. Science. 2017;356(6335):eaah4573.

36. Rhodes J, et al. Human dendritic cell subsets, ontogeny, and impact on HIV infection. Front Immunol. 2019;10:1088.

37. Collin M, Bigley V. Human dendritic cell subsets: an update. Immunology. 2018;154(1):3-20.

38. Calzetti F, et al. Human dendritic cell subset 4 (DC4) correlates to a subset of $\mathrm{CD} 14^{\mathrm{dim} /}-\mathrm{CD} 16^{++}$monocytes. JAllergy Clin Immunol. 2018;141(6):2276-2279.

39. Bose DA, et al. RNA binding to CBP stimulates histone acetylation and transcription. Cell. 2017;168(1-2):135-149.

40. Kuo C-C, et al. Detection of RNA-DNA binding sites in long noncoding RNAs. Nucleic Acids Res. 2019;47(6):e32.

41. Skene PJ, et al. Targeted in situ genome-wide profiling with high efficiency for low cell numbers. Nat Protoc. 2018;13(5):1006-1019.

42. Snyder JP, Amiel E. Regulation of dendritic cell immune function and metabolism by cellular nutrient sensor mammalian target of rapamycin (mTOR). Front Immunol. 2019;9:3145.

43. Altfeld M, Gale M Jr. Innate immunity against HIV-1 infection. Nat Immunol. 2015;16(6):554-562.

44. Espíndola MS, et al. HIV infection: focus on the innate immune cells. Immunol Res. 2016;64(56):1118-1132.

45. Bergantz L, et al. Interplay between Intrinsic and Innate Immunity during HIV Infection. Cells. 2019;8(8):922.

46. Gaiha GD, et al. Structural topology defines protective $\mathrm{CD} 8^{+} \mathrm{T}$ cell epitopes in the HIV proteome. Science. 2019;364(6439):480-484.

47. Marras F, et al. Natural killer cells in HIV controller patients express an activated effector phenotype and do not up-regulate NKp44 on IL-2 stimulation. Proc Natl Acad Sci U S A. 2013;110(29):11970-11975.

48. May ME, et al. Combined effects of HLAB*57/5801 elite suppressor CD8+ T cells and NK cells on HIV-1 replication. Front Cell Infect Microbiol. 2020;10:113.

49. Li H, et al. Short communication: plasmacytoid dendritic cells from HIV-1 elite controllers main- tain a gut-homing phenotype associated with immune activation. AIDS Res Hum Retroviruses. 2014;30(12):1213-1215.

50. Machmach K, et al. Plasmacytoid dendritic cells reduce HIV production in elite controllers. J Virol. 2012;86(8):4245-4252.

51. Herbeuval JP, et al. Characteristics of plasmacytoid dendritic cell and CD4+ T cell in HIV elite controllers. Clin Dev Immunol. 2012;2012:869505.

52. Huang J, et al. Leukocyte immunoglobulin-like receptors maintain unique antigen-presenting properties of circulating myeloid dendritic cells in HIV-1-infected elite controllers. J Virol. 2010;84(18):9463-9471.

53. Hamimi C, et al. Dendritic cells from HIV controllers have low susceptibility to HIV-1 infection in vitro but high capacity to capture HIV-1 particles. PLoS One. 2016;11(8):e0160251.

54. Dong X, et al. Long non-coding RNA MIR4435$2 \mathrm{HG}$ promotes colorectal cancer proliferation and metastasis through miR-206/YAP1 Axis. Front Oncol. 2020;10:160.

55. Qian H, et al. The IncRNA MIR4435-2HG promotes lung cancer progression by activating $\beta$-catenin signalling. J Mol Med (Berl). 2018;96(8):753-764.

56. Angin M, et al. Metabolic plasticity of HIV-specific $\mathrm{CD}^{+} \mathrm{T}$ cells is associated with enhanced antiviral potential and natural control of HIV-1 infection. Nat Metab. 2019;1(7):704-716.

57. Sáez-Cirión A, Sereti I. Immunometabolism and HIV-1 pathogenesis: food for thought. Nat Rev Immunol. 2020;21(1):5-19.

58. Valle-Casuso J, et al. Cellular metabolism is a major determinant of HIV-1 reservoir seeding in $\mathrm{CD} 4^{+} \mathrm{T}$ cells and offers an opportunity to tackle infection. Cell Metab. 2019;29(3):611-626.

59. Saravia J, et al. Signaling networks in immunometabolism. Cell Res. 2020;30(4):328-342.

60. Malinarich F, et al. High mitochondrial respiration and glycolytic capacity represent a metabolic phenotype of human tolerogenic dendritic cells. JImmunol. 2015;194(11):5174-5186.

61. Carroll RG, et al. Immunometabolism around the clock. Trends Mol Med.2019;25(7):612-625.

62. Everts B, Pearce EJ. Metabolic control of dendritic cell activation and function: recent advances and clinical implications. Front Immunol. 2014;5:203.

63. O'Neill L, Pearce EJ. Immunometabolism governs dendritic cell and macrophage function. JExp Med. 2016;213(1):15-23.

64. Saeed S, et al. Epigenetic programming of monocyte-to-macrophage differentiation and trained innate immunity. Science. 2014;345(6204):1251086-1251086.

65. Cheng SC, et al. mTOR- and HIF-1 -mediated aerobic glycolysis as metabolic basis for trained immunity. Science. 2014;345(6204):12506841250684

66. Arts R, et al. Immunometabolic circuits in trained immunity. Semin Immunol. 2016;28(5):425-430.

67. Arts R, et al. BCG vaccination protects against experimental viral infection in humans through the induction of cytokines associated with trained immunity. Cell Host Microbe. 2018;23(1):89-100.

68. Netea MG, et al. Trained immunity: a tool for reducing susceptibility to and the severity of SARS-CoV-2 infection. Cell. 2020;181(5):969-977.

69. Priem B, et al. Trained immunity-promoting nanobiologic therapy suppresses tumor growth and potentiates checkpoint inhibition. Cell. 2020;183(3):786-801.

70. Pereyra F, et al. The major genetic determinants of HIV-1 control affect HLA class I peptide presentation. Science. 2010;330(6010):1551-1557.

71. Trombetta JJ, et al. Preparation of single-cell RNA-seq libraries for next generation sequencing. Curr Protoc Mol Biol. 2014;107:4.22.1-17.

72. Dobin A, et al. STAR: ultrafast universal RNA-seq aligner. Bioinformatics. 2013;29(1):15-21.

73. Li B, Dewey CN. RSEM: accurate transcript quantification from RNA-Seq data with or without a reference genome. BMC Bioinformatics. 2011;12(1):323.

74. Risso D, et al. Normalization of RNA-seq data using factor analysis of control genes or samples. Nat Biotechnol. 2014;32(9):896-902.

75. Love MI, et al. Moderated estimation of fold change and dispersion for RNA-seq data with DESeq2. Genome Biol. 2014;15(12):550.

76. Bolger AM, et al. Trimmomatic: a flexible trimmer for Illumina sequence data. Bioinformatics. 2014;30(15):2114-2120

77. Langmead B, Salzberg SL. Fast gappedread alignment with Bowtie 2. Nat Methods. 2012;9(4):357-359.

78. Zhang Y, et al. Model-based analysis of ChIP-Seq (MACS). Genome Biol. 2008;9(9):R137.

79. Ramírez F, et al. deepTools2: a next generation web server for deep-sequencing data analysis. Nucleic Acids Res. 2016;44(W1):5-W165.

80. Ross-Innes CS, et al. Differential oestrogen receptor binding is associated with clinical outcome in breast cancer. Nature. 2012;481(7381):389-393. 
